\title{
LANDAU LEVELS IN A TWO-DIMENSIONAL NONCOMMUTATIVE SPACE: MATRIX AND QUATERNIONIC VECTOR COHERENT STATES
}

\author{
MAHOUTON NORBERT HOUNKONNOU ${ }^{*, \dagger}$ \\ and ISIAKA AREMUA ${ }^{\ddagger}$ \\ International Chair in Mathematical Physics \\ and Applications, ICMPA-UNESCO Chair \\ University of Abomey-Calavi \\ 072 B.P. 50 Cotonou, Republic of Benin \\ *norbert.hounkonnou@cipma.uac.bj \\ †hounkonnou@yahoo.fr \\ ${ }^{\ddagger}$ claudisak@yahoo.fr
}

Received 7 July 2012

Accepted 29 August 2012

Published 31 December 2012

\begin{abstract}
The behavior of an electron in an external uniform electromagnetic background coupled to an harmonic potential, with noncommuting space coordinates, is considered in this work. The thermodynamics of the system is studied. Matrix vector coherent states (MVCS) as well as quaternionic vector coherent states (QVCS), satisfying required properties, are also constructed and discussed.
\end{abstract}

Keywords: Noncommutative space; quantum Hilbert space; vector coherent state.

\section{Introduction}

Noncommuting spatial coordinates and fields can (approximately) be realized in actual physical situations. Landau models and their quantum Hall limit have become the focus of intense research activity as a physical realization of the simplest example of noncommutative geometry [7, 8, 14-17, 19, 27, 29-31, 35-38, 43, 46-48, 53]. Similar structures also arise in specific approaches towards a theory of quantum gravity, such as $M$-theory in the presence of background fields [52] or tentative formulations of relativistic quantum theories of gravity through spacetime noncommutativity. ${ }^{\text {a }}$

As is well known [29], given a point particle of mass $m$, charge $\bar{q}$ and position $\vec{r}=(x, y)$ moving in a plane in the presence of a constant external magnetic field $B$ perpendicular to that plane, the spectrum of the quantized theory is organized into infinitely degenerate Landau levels, with separation $\mathcal{O}(\bar{q} B / m)$. The limit $B \rightarrow \infty$ effectively projects onto the

\footnotetext{
${ }^{\mathrm{a}}$ For recent material on Noncommutativity and Quantum Gravity, see for instance [39].
} 
lowest Landau level and is equivalent to a negligibly small mass $m$, i.e. $m \rightarrow 0$. Consequently in each of the projected Landau levels, one obtains a noncommuting algebra for the space coordinates,

$$
[x, y]=-\frac{i \hbar}{\bar{q} B}
$$

Historically, this is the Peierls substitution rule introduced seventy years ago [42]. As a matter of fact, this noncommuting character arises already at the classical level in terms of the Dirac brackets associated to the second-class constraints that follow upon taking the limit $m \rightarrow 0$ in the classical Hamiltonian formulation of the dynamics of the Landau problem and requiring finite energy configurations.

Given the simplicity and ubiquity of the above two-dimensional system in problems involving background fields, as is the case for instance in $M$-theory and its many $D$-brane constructions [52], the Landau problem and its noncommutative quantum Hall limit have played a central role in recent years as inspiration and guide towards tentative formulation of relativistic quantum theories of gravity through spacetime noncommutativity.

Besides, in most of the introductory references in the literature devoted to quantum mechanics and quantum field theory, it comes out that the natural appearance of noncommutativity in string theories has increasingly led to attempts to study physical problems in noncommutative spaces $[11,12]$. Although noncommuting coordinates are operators even at the classical level, one can treat them as commuting by replacing operator products by $*$-products [9]. This approach allows one to generalize classical as well as quantum mechanics without altering their main physical interpretations and to recover the usual results when noncommutativity is switched off. In some recent works, the quantum Hall system has attracted considerable attention from the point of view of noncommutative quantum mechanics and quantum field theory (see e.g. $[10,16,17,27,41]$ ). Note that a noncommutative model valid for a constant magnetic field, with respect to the geometrical aspect of the problem, has been also investigated. For more details, see [40]. The description of such a system $[24,25]$ is adequately provided by the well known Landau model [33] as mentioned above. This latter describes the motion of an electron in a uniform magnetic field which can be assimilated to that of a two-dimensional harmonic oscillator. Since this discovery, the quantum states of a particle in a magnetic and electromagnetic fields on noncommutative plane have been attracting considerable attention, see for instance $[3,10,13,16,17,19,21,22,27,32]$ and more recently [56] (and references listed therein). In [51], based on a previous work [49], the thermodynamics of an ideal fermion gas confined in a two-dimensional noncommutative well has been investigated. The authors have shown that the thermodynamical properties of the fermion gas for the commutative and noncommutative cases agree at low densities, while at high densities they start diverging strongly due to the implied excluded area resulting from the noncommutativity. In [20] the possible occurrence of orbital magnetism for two-dimensional electrons confined by an harmonic potential [28] in various regimes of temperature and magnetic field has been studied. Coherent states (CS) have been applied to investigations on noncommutative geometries [26]. One of their primary properties is the minimization of the position and momentum uncertainty relation. They are also relevant for studying the Dirac operator in fuzzy quantum spaces, when the underlying spacetime or spatial cut can be treated as a phase space 
and quantized, as demonstrated in [1]. Besides, they have been formulated in terms of the diagonal coherent state matrix elements of operators and star products [4] in the analysis of models implying noncommutative Chern-Simons theory. Standard coherent states have been used to calculate symbols of various observables like the thermodynamical potential, the magnetic moment or the spatial distribution of the current in the system. In [32], an analogous treatment in a noncommutative framework has been achieved and the results of $[20]$ in the commutative case have been recovered by switching off the $\theta$-parameter.

In the noncommutative quantum mechanics formulation, a major role is played by the CS on the quantum Hilbert space denoted by $\mathcal{H}_{q}$ (the space of Hilbert Schmidt operators on the classical configuration space denoted by $\mathcal{H}_{c}$ ), which are expressed in terms of a projection operator on the usual Glauber-Klauder-Sudarshan CS in the classical configuration space. Based on the approach developed in [50], Gazeau-Klauder CS have been constructed in the noncommutative quantum mechanics [23]. These states share similar properties to those of ordinary canonical CS in the sense that they saturate the related position uncertainty relation, obey a Poisson distribution and possess a flat geometry. The thermodynamics of an ideal fermion gas has been investigated in [51].

This work deals with the study of the electron motion in an external uniform electromagnetic field, (the so-called Landau problem), coupled with an harmonic potential in a two-dimensional noncommutative space. The thermodynamics of this physical system is investigated following the method established in [20] by formulating at first CS on the quantum Hilbert space $\mathcal{H}_{q}$. Then, relevant inequalities are deduced and used to compute both the thermodynamical potential and magnetic moment. Besides, we complete this study with the construction of vector cs (VCS) and show that they fulfill a resolution of the identity on a suitable Hilbert space. This construction fits the general formulation of VCS by Ali et al. [2] instead of the general theory of vector-valued coherent state representations [45] (and references listed therein) and similar developments based on operators of unitary representations of groups, where the VCS are defined as orbits of vectors [5, 6, 44]. Furthermore, we extend the VCS construction used in [23] to a formal tensor product of quantum Hilbert spaces by using the primary formulation of [55]. This extension is implemented with complex matrices and quaternions as CS variables. The physical features of the quaternionic VCS (QVCS) are discussed. The expectation values and dispersions of the quadrature operators are provided. The constructed QVCS reveal some inequalities inherited from the parametrization and the noncommutativity of the space coordinates.

The paper is organized as follows. In Sec. 2, we describe the physical model and give the corresponding matrix formulation. The Hamiltonian spectrum and its spectral decomposition are provided. The definition of the passage operators from an orthonormal basis to another one is also supplied. Section 3 discusses the thermodynamical aspects of the studied model. In Sec. 4, relevant VCS and QVCS for Landau levels are constructed and analysed. Finally, there follow concluding remarks in Sec. 5.

\section{The Electron in Noncommutative Plane}

\subsection{Quantum model}

The physics of an electron in crossed constant uniform electric $\mathbf{E}$ and magnetic $\mathbf{B}$ fields coupled with a confining harmonic potential in a noncommutative space, is described, in 
the gauge $\mathbf{A}=\left(-\frac{B}{2} y, \frac{B}{2} x\right)$, by the Hamiltonian:

$$
H_{\theta}=\frac{1}{2 M}\left(\hat{P}_{i}-\frac{e B}{2 c} \epsilon_{i j} \hat{X}_{j}\right)^{2}+\frac{M \omega_{0}^{2}}{2} \hat{X}_{i}^{2}-e E_{i} \hat{X}_{i}, \quad \epsilon_{j i}=-\epsilon_{i j}, \quad \epsilon_{12}=+1,
$$

where the position and momentum operators $\hat{X}_{i}=\hat{X}, \hat{Y}$ and $\hat{P}_{i}=\hat{P}_{X}, \hat{P}_{Y}, i=1,2$, satisfy the following commutation relations of the noncommutative Heisenberg algebra [50]:

$$
[\hat{X}, \hat{Y}]=i \theta, \quad\left[\hat{X}, \hat{P}_{X}\right]=i \hbar=\left[\hat{Y}, \hat{P}_{Y}\right], \quad\left[\hat{P}_{X}, \hat{P}_{Y}\right]=0, \quad\left[\hat{X}, \hat{P}_{Y}\right]=0=\left[\hat{Y}, \hat{P}_{X}\right]
$$

The position operators $\hat{X}_{i}$ and their corresponding canonically conjugate momenta $\hat{P}_{i}$ can be combined in the operators $\hat{\Pi}_{i}=\hat{P}_{i}-\frac{e B}{2 c} \epsilon_{i j} \hat{X}_{j}$ to yield the relations:

$$
\left[\hat{X}_{i}, \hat{\Pi}_{j}\right]=i\left(\hbar-\frac{e B}{2 c} \theta\right) \delta_{i j}, \quad\left[\hat{\Pi}_{i}, \hat{\Pi}_{j}\right]=-i \frac{e B}{c}\left(\hbar-\frac{e B}{4 c} \theta\right) \epsilon_{i j} .
$$

From the latters, define the complex canonically conjugate momenta, denoted by $\hat{\Pi}_{Z}$ corresponding to $\hat{Z}=\hat{X}+i \hat{Y}$ and $\hat{\bar{Z}}=\hat{X}-i \hat{Y}$ by

$$
\hat{\Pi}_{Z}=\hat{\Pi}_{X}-i \hat{\Pi}_{Y}, \quad \hat{\Pi}_{\bar{Z}}=\hat{\Pi}_{X}+i \hat{\Pi}_{Y}
$$

respectively, such that the quantum operators $\hat{Z}, \hat{\bar{Z}}, \hat{\Pi}_{Z}, \hat{\Pi}_{\bar{Z}}$ act on the quantum Hilbert space $\mathcal{H}_{q}[21,50]$, i.e. the space of Hilbert-Schmidt operators acting on the noncommutative configuration (Hilbert) space $\mathcal{H}_{c}$, defined as:

$$
\mathcal{H}_{q}=\left\{\psi(\hat{z}, \hat{\bar{z}}): \psi(\hat{z}, \hat{\bar{z}}) \in \mathcal{B}\left(\mathcal{H}_{c}\right), \operatorname{tr}_{c}\left(\psi(\hat{z}, \hat{\bar{z}})^{\dagger}, \psi(\hat{z}, \hat{\bar{z}})\right)<\infty\right\}
$$

endowed with the following inner product

$$
\left(\psi\left(\hat{x}_{1}, \hat{x}_{2}\right), \phi\left(\hat{x}_{1}, \hat{x}_{2}\right)\right)=\operatorname{tr}_{c}\left(\psi\left(\hat{x}_{1}, \hat{x}_{2}\right)^{\dagger}, \phi\left(\hat{x}_{1}, \hat{x}_{2}\right)\right),
$$

where $\operatorname{tr}_{c}$ stands for the trace over $\mathcal{H}_{c} \cdot \mathcal{B}\left(\mathcal{H}_{c}\right)$ is the set of bounded operators on $\mathcal{H}_{c}$.

Remark 2.1. For an harmonic oscillator, the two-dimensional noncommutative coordinate algebra is given by

$$
[\hat{x}, \hat{y}]=i \theta
$$

$\theta$ refers to as the noncommutativity parameter. The annihilation and creation operators $a=1 / \sqrt{2 \theta}(\hat{x}+i \hat{y}), a^{\dagger}=1 / \sqrt{2 \theta}(\hat{x}-i \hat{y})$ obey a Heisenberg-Fock algebra $\left[a, a^{\dagger}\right]=1_{c}$, where $1_{c}$ is the identity operator on the Hilbert space $\mathcal{H}_{c}$, i.e. the noncommutative configuration space which becomes itself a Hilbert space isomorphic to the boson Fock space [50] $\mathcal{H}_{c}=$ $\operatorname{span}\{|n\rangle, n \in \mathbb{N}\}$, with $|n\rangle=1 / \sqrt{n !}\left(a^{\dagger}\right)^{n}|0\rangle$.

As mentioned in [23], a well defined representation with self-adjoint properties with respect to the quantum Hilbert space $\mathcal{H}_{q}$ inner product is provided by the following relations

$$
\hat{X} \psi=\hat{x} \psi, \quad \hat{Y} \psi=\hat{y} \psi, \quad \hat{P}_{X} \psi=\frac{\hbar}{\theta}[\hat{y}, \psi], \quad \hat{P}_{Y} \psi=-\frac{\hbar}{\theta}[\hat{x}, \psi] .
$$


On the Hilbert space $\mathcal{H}_{q}$, the following commutation relations are satisfied:

$$
\begin{gathered}
{[\hat{Z}, \hat{\bar{Z}}]=2 \theta, \quad\left[\hat{Z}, \hat{\Pi}_{Z}\right]=2 i\left(\hbar-\frac{e B}{2 c} \theta\right)=\left[\hat{\bar{Z}}, \hat{\Pi}_{\bar{Z}}\right]} \\
{\left[\hat{\Pi}_{Z}, \hat{\Pi}_{\bar{Z}}\right]=2 \frac{e B}{c}\left(\hbar-\frac{e B}{4 c} \theta\right), \quad\left[\hat{Z}, \hat{\Pi}_{\bar{Z}}\right]=0=\left[\hat{\bar{Z}}, \hat{\Pi}_{Z}\right] .}
\end{gathered}
$$

For the analysis purpose, defining a diagonal matrix $\mathcal{D}$ and adopting the notations $E=\left(E_{1}, E_{2}, 0,0\right), \mathcal{X}_{0}=\left(x_{0}, y_{0}, 0,0\right)^{t}$, where $x_{0}=\frac{e E_{1}}{M \omega_{0}^{2}}, y_{0}=\frac{e E_{2}}{M \omega_{0}^{2}}$, the Hamiltonian $H_{\theta}$ of the physical model can be rewritten in a short form as follows:

$$
H_{q}=\frac{1}{4 M} \hat{\mathcal{Z}}^{\ddagger} \hat{\mathcal{Z}}-\frac{1}{2} e E \cdot \mathcal{X}_{0}=\frac{1}{4 M} A^{\ddagger} \mathcal{D} A-\frac{1}{2} e E \cdot \mathcal{X}_{0}, \quad A=\left(B_{+}, B_{+}^{\ddagger}, B_{-}, B_{-}^{\ddagger}\right),
$$

where the notation $\ddagger$ is reserved to denote the Hermitian conjugation on the quantum Hilbert space, the annihilation $B_{ \pm}$and creation $B_{ \pm}^{\ddagger}$ operators defined on the helicity quantum Hilbert space as

$$
\begin{array}{ll}
B_{+}=\zeta \frac{\hat{\bar{Z}}-\bar{Z}_{0}}{2}+\frac{i}{\zeta \hbar}\left(\hat{P}_{Z}-P_{Z_{0}}\right), & B_{+}^{\ddagger}=\zeta \frac{\hat{Z}-Z_{0}}{2}-\frac{i}{\zeta \hbar}\left(\hat{P}_{\bar{Z}}-P_{\bar{Z}_{0}}\right), \\
B_{-}=\zeta \frac{\hat{Z}-Z_{0}}{2}+\frac{i}{\zeta \hbar}\left(\hat{P}_{\bar{Z}}-P_{\bar{Z}_{0}}\right), & B_{-}^{\ddagger}=\zeta \frac{\hat{\bar{Z}}-\bar{Z}_{0}}{2}-\frac{i}{\zeta \hbar}\left(\hat{P}_{Z}-P_{Z_{0}}\right),
\end{array}
$$

with $\hat{P}_{Z}=\frac{1}{2}\left(\hat{P}_{X}-i \hat{P}_{Y}\right), \hat{P}_{\bar{Z}}=\frac{1}{2}\left(\hat{P}_{X}+i \hat{P}_{Y}\right)$ verifying

$$
\left[\hat{Z}, \hat{P}_{Z}\right]=i \hbar=\left[\hat{\bar{Z}}, \hat{P}_{\bar{Z}}\right], \quad\left[\hat{Z}, \hat{P}_{\bar{Z}}\right]=0=\left[\hat{\bar{Z}}, \hat{P}_{Z}\right], \quad\left[\hat{P}_{Z}, \hat{P}_{\bar{Z}}\right]=0 .
$$

The quantities

$$
Z_{0}=x_{0}+i y_{0}, \quad P_{Z_{0}}=\frac{1}{2}\left(p_{x_{0}}-i p_{y_{0}}\right), \quad p_{x_{0}}=-\frac{e B}{2 c} y_{0}, \quad p_{y_{0}}=\frac{e B}{2 c} x_{0},
$$

are such that the annihilation $B_{ \pm}$and creation $B_{ \pm}^{\ddagger}$ operators satisfy the commutation relations:

$$
\left[B_{ \pm}, B_{ \pm}^{\ddagger}\right]=1_{q}, \quad\left[B_{ \pm}, B_{\mp}^{\ddagger}\right]=0, \quad\left[B_{+}, B_{-}\right]=0, \quad\left[B_{+}^{\ddagger}, B_{-}^{\ddagger}\right]=0 .
$$

Now introduce the operators

$$
\begin{aligned}
& A^{+}=\left(B_{+}^{\ddagger}, B_{+}, B_{-}^{\ddagger}, B_{-}\right)^{t}=\Lambda A, \\
& \hat{\mathcal{Z}}^{+}=\left(\hat{\bar{Z}}^{\prime}-\bar{Z}_{0}^{\prime}, \hat{Z}^{\prime}-Z_{0}^{\prime}, \hat{\Pi}_{\bar{Z}}-\Pi_{\bar{Z}_{0}}, \hat{\Pi}_{Z}-\Pi_{Z_{0}}\right)^{t}=\Lambda \hat{\mathcal{Z}},
\end{aligned}
$$

with

$$
\Pi_{Z_{0}}=\Pi_{X_{0}}-i \Pi_{Y_{0}}, \quad \Pi_{X_{0}}=p_{x_{0}}-\frac{e B}{2 c} y_{0}, \quad \Pi_{Y_{0}}=p_{y_{0}}+\frac{e B}{2 c} x_{0},
$$

where $\hat{Z}^{\prime}-Z_{0}^{\prime}=M \omega_{0}\left(\hat{Z}-Z_{0}\right)$, with the permutation matrix $\Lambda$ defined by

$$
\Lambda=\left(\begin{array}{llll}
0 & 1 & 0 & 0 \\
1 & 0 & 0 & 0 \\
0 & 0 & 0 & 1 \\
0 & 0 & 1 & 0
\end{array}\right)
$$


The symbol $t$ means the transpose operation.

Then, consider the matrix $\mathfrak{g}$ with entries $\mathfrak{g}_{l k}=\left[\hat{\mathcal{Z}}_{l}, \hat{\mathcal{Z}}_{k}^{+}\right], l, k=1, \ldots, 4$, obtained from the commutation relations (2.9) as follows:

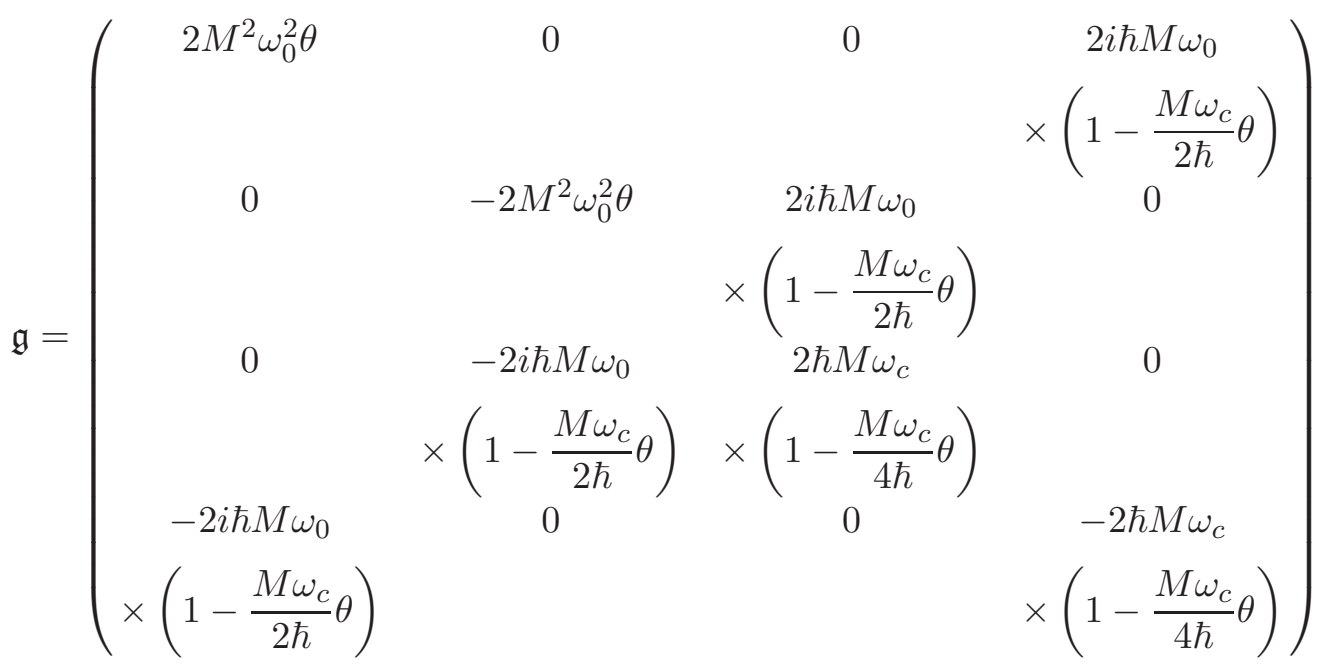

with the eigenvalues $\tilde{\lambda}_{ \pm},-\tilde{\lambda}_{ \pm}$supplied by the expressions

$$
\tilde{\lambda}_{ \pm}=M \hbar\left\{\Omega \sqrt{1-\frac{M \omega_{c}}{2 \hbar} \theta+\left(\frac{M \Omega}{2 \hbar} \theta\right)^{2}} \pm \omega_{c}\left(1-\left(\frac{\omega_{c}}{4 \hbar}+\frac{\omega_{0}^{2}}{\hbar \omega_{c}}\right) M \theta\right)\right\}
$$

where $\Omega^{2}=4 \omega_{0}^{2}+\omega_{c}^{2}$. The matrix $\mathcal{S}^{\dagger}$, eigenvector matrix of $\mathfrak{g}$, is given by

$$
\mathcal{S}^{\dagger}=\left(\frac{1}{\sqrt{\left|\lambda_{+}\right|}} u_{1}^{\prime}, \frac{1}{\sqrt{\left|\lambda_{+}\right|}}\left(\Lambda u_{1}^{*}\right)^{\prime}, \frac{1}{\sqrt{\left|\lambda_{-}\right|}} u_{2}^{\prime}, \frac{1}{\sqrt{\left|\lambda_{-}\right|}}\left(\Lambda u_{2}^{*}\right)^{\prime}\right) .
$$

The normalized eigenvectors $\left(u_{1}^{\prime},\left(\Lambda u_{1}^{*}\right)^{\prime}\right)$ and $\left(u_{2}^{\prime},\left(\Lambda u_{2}^{*}\right)^{\prime}\right)$ associated with $\left(\tilde{\lambda}_{+},-\tilde{\lambda}_{+}\right)$and $\left(\tilde{\lambda}_{-},-\tilde{\lambda}_{-}\right)$, respectively, are provided by

$$
u_{1}^{\prime}=\frac{1}{\left\|u_{1}\right\|} u_{1}, \quad\left(\Lambda u_{1}^{*}\right)^{\prime}=\frac{1}{\left\|\Lambda u_{1}^{*}\right\|}\left[\Lambda u_{1}^{*}\right], \quad u_{2}^{\prime}=\frac{1}{\left\|u_{2}\right\|} u_{2}, \quad\left(\Lambda u_{2}^{*}\right)^{\prime}=\frac{1}{\left\|\Lambda u_{2}^{*}\right\|}\left[\Lambda u_{2}^{*}\right],
$$

with

$$
u_{1}=\left(\begin{array}{c}
0 \\
i \frac{B_{\hbar}}{\kappa_{+}} \\
1 \\
0
\end{array}\right), \quad u_{2}=\left(\begin{array}{c}
i \frac{B_{\hbar}}{\kappa_{-}} \\
0 \\
0 \\
1
\end{array}\right)
$$

$u_{j}^{*}, j=1,2$, are the vectors with entries which are conjugate to the $u_{j}$ entries; $B_{\hbar}=$ $2 \hbar M \omega_{0}\left(1-\frac{M \omega_{c}}{2 \hbar} \theta\right)$ and

$$
\kappa_{ \pm}=M \hbar\left\{\Omega \sqrt{1-\frac{M \omega_{c}}{2 \hbar} \theta+\left(\frac{M \Omega}{4 \hbar} \theta\right)^{2}} \pm \omega_{c}\left(1-\left(\frac{\omega_{c}}{4 \hbar}-\frac{\omega_{0}^{2}}{\hbar \omega_{c}}\right) M \theta\right)\right\} .
$$


Then, the Hamiltonian is obtained as

$$
H_{q}=\frac{1}{4 M} A^{\ddagger} \mathcal{D} A-\frac{1}{2} e E . \mathcal{X}_{0}=\frac{1}{4 M} A^{\ddagger} \mathbb{J}_{4} \mathcal{S}^{2} \mathcal{S}^{\dagger} \mathbb{J}_{4} A-\frac{1}{2} e E . \mathcal{X}_{0},
$$

where $\mathbb{J}_{4}$ is given by

$$
\mathbb{J}_{4}=\operatorname{diag}\left(\sigma_{3}, \sigma_{3}\right), \quad \sigma_{3}=\left(\begin{array}{rr}
1 & 0 \\
0 & -1
\end{array}\right)
$$

The $\theta$-dependent quantities $\tilde{\Omega}, \tilde{\omega}_{c}$ and $\zeta$ introduced in (2.11) are determined as

$$
\begin{aligned}
& \tilde{\Omega}=\Omega \sqrt{1-\frac{M \omega_{c}}{2} \theta+\left(\frac{M \Omega}{4} \theta\right)^{2}}, \quad \tilde{\omega}_{c}=\omega_{c}\left(1-\left(\frac{\omega_{c}}{4}+\frac{\omega_{0}^{2}}{\omega_{c}}\right) M \theta\right) \\
& \zeta=\sqrt{\frac{M \Omega}{\hbar}} \frac{1}{\mu_{\theta}}=\sqrt[4]{\frac{(M \Omega / \hbar)^{2}}{1-\frac{M \omega_{c}}{2} \theta+\left(\frac{M \Omega}{4} \theta\right)^{2}}}, \quad \mu_{\theta}=\sqrt[4]{1-\frac{M \omega_{c}}{2} \theta+\left(\frac{M \Omega}{4} \theta\right)^{2}} .
\end{aligned}
$$

Set $\tilde{\Omega}_{ \pm}=\frac{\tilde{\Omega} \pm \tilde{\omega}_{c}}{2}$. The Hamiltonian $H_{q}$ is re-expressed in terms of positive quantities $\tilde{\Omega}_{ \pm}$ as follows:

$$
H_{q}=\hbar\left(\tilde{\Omega}_{+} \tilde{N}_{+}+\tilde{\Omega}_{-} \tilde{N}_{-}+\frac{\tilde{\Omega}}{2}\right)-\frac{1}{2} e\left(E_{1} x_{0}+E_{2} y_{0}\right),
$$

where $\tilde{N}_{ \pm}=B_{ \pm}^{\ddagger} B_{ \pm}$denote the number operators on the quantum Hilbert space.

Finally, there result the eigenvalues of the Hamiltonian $H_{q}$, expressed in the Fock helicity representation $\left|\tilde{n}_{+}, \tilde{n}_{-}\right\rangle$by

$$
E_{\tilde{n}_{+}, \tilde{n}_{-}}=\hbar\left(\tilde{\Omega}_{+} \tilde{n}_{+}+\tilde{\Omega}_{-} \tilde{n}_{-}+\frac{\tilde{\Omega}}{2}\right)-\frac{1}{2} e\left(E_{1} x_{0}+E_{2} y_{0}\right)
$$

with the corresponding eigenvectors on the quantum Hilbert space given by

$$
\left.\mid \tilde{n}_{+}, \tilde{n}_{-}\right)=\frac{1}{\sqrt{\tilde{n}_{+} ! \tilde{n}_{-} !}}\left(B_{+}^{\ddagger}\right)^{\tilde{n}_{+}}\left(B_{-}^{\ddagger}\right)^{\tilde{n}_{-}}|0\rangle\langle 0|,
$$

where $B_{-}^{\ddagger}$ may have an action on the right by $B_{-}$on $|0\rangle\langle 0|$ which stands for the vacuum state on $\mathcal{H}_{q}$ and $\left.\| \mid \tilde{n}_{+}, \tilde{n}_{-}\right) \|=1$.

The annihilation and creation operators act on the states $\left.\mid \tilde{n}_{+}, \tilde{n}_{-}\right)=\left|\tilde{n}_{+}\right\rangle\left\langle\tilde{n}_{-}\right|, \tilde{n}_{ \pm}=$ $0,1,2, \ldots$, as follows:

$$
\begin{aligned}
& \left.\left.\left.\left.B_{+} \mid \tilde{n}_{+}, \tilde{n}_{-}\right)=\sqrt{\tilde{n}_{+}} \mid \tilde{n}_{+}-1, \tilde{n}_{-}\right), \quad B_{+}^{\ddagger} \mid \tilde{n}_{+}, \tilde{n}_{-}\right)=\sqrt{\tilde{n}_{+}+1} \mid \tilde{n}_{+}+1, \tilde{n}_{-}\right), \\
& \left.\left.\left.\left.B_{-} \mid \tilde{n}_{+}, \tilde{n}_{-}\right)=\sqrt{\tilde{n}_{-}} \mid \tilde{n}_{+}, \tilde{n}_{-}-1\right), \quad B_{-}^{\ddagger} \mid \tilde{n}_{+}, \tilde{n}_{-}\right)=\sqrt{\tilde{n}_{-}+1} \mid \tilde{n}_{+}, \tilde{n}_{-}+1\right) .
\end{aligned}
$$




\subsection{Spectral decomposition}

Let us consider the dimensionless shifted quantum Hamiltonian

$$
H_{q}^{\operatorname{dim}}=\frac{1}{\hbar \tilde{\Omega}}\left[H_{q}+\frac{1}{2} e\left(E_{1} x_{0}+E_{2} y_{0}\right)\right]
$$

with associated eigenvalues

$$
\tilde{E}_{\tilde{n}_{+}, \tilde{n}_{-}}=\frac{\tilde{\Omega}_{+}}{\tilde{\Omega}} \tilde{n}_{+}+\frac{\tilde{\Omega}_{-}}{\tilde{\Omega}} \tilde{n}_{-}+\frac{1}{2} .
$$

Take $\left.\left\{\mid \tilde{n}_{+}, \tilde{n}_{-}\right), \tilde{n}_{ \pm} \in \mathbb{N}\right\}$ as the orthonormal eigenstate basis associated with the quantum Hamiltonian $H_{q}$ in the helicity Fock algebra representation. With respect to the inner product on $\mathcal{H}_{q}$, we have $\left(\tilde{n}_{+}, \tilde{n}_{-} \mid \tilde{n}_{+}^{\prime}, \tilde{n}_{-}^{\prime}\right)=\operatorname{tr}_{c}\left[\left(\left|\tilde{n}_{+}\right\rangle\left\langle\tilde{n}_{-}\right|\right)^{\ddagger}\left|\tilde{n}_{+}^{\prime}\right\rangle\left\langle\tilde{n}_{-}^{\prime}\right|\right]=\delta_{\tilde{n}_{+}, \tilde{n}_{+}^{\prime}} \delta_{\tilde{n}_{-}, \tilde{n}_{-}^{\prime}}$. On this basis, the Hamiltonian $H_{q}^{\text {dim }}$ admits the following spectral decomposition

$$
\left.H_{q}^{\operatorname{dim}}=\sum_{\tilde{n}_{ \pm}=0}^{\infty} \mid \tilde{n}_{+}, \tilde{n}_{-}\right) \tilde{E}_{\tilde{n}_{+}, \tilde{n}_{-}}\left(\tilde{n}_{+}, \tilde{n}_{-} \mid\right.
$$

Let $\{|n\rangle\langle m|:=| n, m), n, m \in \mathbb{N}\}$ be the orthonormal basis associated with the quantum Hilbert space $\mathcal{H}_{q}$. Introduce the passage operators from $\left.\{\mid n, m), n, m \in \mathbb{N}\right\}$ to $\left.\left\{\mid \tilde{n}_{+}, \tilde{n}_{-}\right), \tilde{n}_{ \pm} \in \mathbb{N}\right\}$ and vice versa given by

$$
\left.\left.\mathcal{U} \mid n, m)=\mid \tilde{n}_{+}, \tilde{n}_{-}\right), \quad \mathcal{V}\left(\tilde{n}_{+}, \tilde{n}_{-}\right)=\mid n, m\right),
$$

where their expansions are determined by

$$
\left.\mathcal{U}=\sum_{n, m=0}^{\infty} \mid \tilde{n}_{+}, \tilde{n}_{-}\right)\left(n, m\left|, \quad \mathcal{V}=\sum_{\tilde{n}_{ \pm}=0}^{\infty}\right| n, m\right)\left(\tilde{n}_{+}, \tilde{n}_{-} \mid\right.
$$

respectively. $\mathcal{U}, \mathcal{V}$ are obtained as mutually adjoint through the following identities satisfied on $\mathcal{H}_{q}$ :

$$
\left.\mathcal{U} \mathcal{V}=\sum_{\tilde{n}_{ \pm}=0}^{\infty} \mid \tilde{n}_{+}, \tilde{n}_{-}\right)\left(\tilde{n}_{+}, \tilde{n}_{-}\left|=\mathbb{I}_{q}, \quad \mathcal{V U}=\sum_{n, m=0}^{\infty}\right| n, m\right)\left(n, m \mid=\mathbb{I}_{q}\right.
$$

where $\mathbb{I}_{q}$ stands for the identity on $\mathcal{H}_{q}$. Then, the Hamiltonian $H_{q}^{\text {dim }}$ can be rewritten in a diagonal form as follows:

$$
\left.\mathbb{H}^{\operatorname{dim}}=\mathcal{V} H_{q}^{\operatorname{dim}} \mathcal{U}=\sum_{n, m=0}^{\infty} \mid n, m\right) \tilde{E}_{n, m}\left(n, m \mid, \quad \tilde{E}_{n, m}=\frac{\tilde{\Omega}_{+}}{\tilde{\Omega}} n+\frac{\tilde{\Omega}_{-}}{\tilde{\Omega}} m+\frac{1}{2} .\right.
$$

\section{Coherent States and Thermodynamics of the Model}

For the Hamiltonian $H_{q}$ with eigenvalues $E_{\tilde{n}_{+}, \tilde{n}_{-}}=\hbar\left(\tilde{\Omega}_{+} \tilde{n}_{+}+\tilde{\Omega}_{-} \tilde{n}_{-}+\frac{\tilde{\Omega}}{2}\right)+k_{e, E}$, $k_{e, E}=-\frac{1}{2} e\left(E_{1} x_{0}+E_{2} y_{0}\right)$, the cs denoted by $\left.\mid z_{ \pm}, \tau\right)$ are defined on the quantum Hilbert 
space $\mathcal{H}_{q}$ as follows:

$$
\begin{aligned}
\left.\mid z_{ \pm}, \tau\right) & =\mathbb{U}(\tau)\left|z_{+}\right\rangle\left\langle z_{-}\right| \\
& =e^{-\frac{1}{2}\left(\left|z_{+}\right|^{2}+\left|z_{-}\right|^{2}\right)} \sum_{\tilde{n}_{+}, \tilde{n}_{-}=0}^{\infty} \frac{z_{+}^{\tilde{n}_{+}} \bar{z}_{-}^{\tilde{n}_{-}}}{\sqrt{\tilde{n}_{+} ! \tilde{n}_{-} !}} e^{-i \tau E_{\tilde{n}_{+}, \tilde{n}_{-}}}\left|\tilde{n}_{+}\right\rangle\left\langle\tilde{n}_{-}\right| .
\end{aligned}
$$

The parameter $\tau$ is introduced such that the states (3.1) fulfill the Gazeau-Klauder axiom of temporal stability relative to the classical time evolution operator $\mathbb{U}(\tau)=e^{-i\left[H_{q}\right] \tau}$. Indeed, we have the following.

Proposition 3.1. These vectors satisfy the following properties:

- Temporal stability

$$
\left.\left.\left.\mathbb{U}(t) \mid z_{ \pm}, \tau\right)=e^{-i\left[H_{q}\right] t} \mid z_{ \pm}, \tau\right)=\mid z_{ \pm}, \tau+t\right) .
$$

- Action identity, also called lower symbol of $H_{q}$,

$$
\check{H}_{q}\left(z_{ \pm}\right)=\left(z_{ \pm}, \tau\left|H_{q}\right| z_{ \pm}, \tau\right)=\hbar\left(\tilde{\Omega}_{+}\left|z_{+}\right|^{2}+\tilde{\Omega}_{-}\left|z_{-}\right|^{2}+\frac{\tilde{\Omega}}{2}\right)+k_{e, E}
$$

- Resolution of the identity

$$
\left.\frac{1}{\pi^{2}} \int_{\mathbb{C}^{2}} \mid z_{ \pm}, \tau\right)\left(z_{ \pm}, \tau \mid d^{2} z_{+} d^{2} z_{-} \equiv \mathbb{I}_{q},\right.
$$

where $\mathbb{I}_{q}$ is the identity operator on $\mathcal{H}_{q}$ provided by

$$
\left.\mathbb{I}_{q}=\frac{1}{\pi} \int_{\mathbb{C}} d z d \bar{z} \mid z\right) e^{\overleftarrow{\partial_{\bar{z}}} \vec{\partial}_{z}}(z \mid
$$

Proof. We have from the definition (3.1) the following relation

$$
\frac{1}{\pi^{2}} \int_{\mathbb{C}^{2}} d^{2} z_{+} d^{2} z_{-}\left|z_{+}\right\rangle\left\langle z_{+}|| z_{-}\right\rangle\left\langle z_{-}\left|\equiv \frac{1}{\pi^{2}} \int_{\mathbb{C}^{2}} d^{2} z_{+} d^{2} z_{-}\right| z_{ \pm}\right)\left(z_{ \pm} \mid,\right.
$$

where

$$
\begin{aligned}
\left.\mid z_{ \pm}\right) & =\left|z_{+}\right\rangle\left\langle z_{-}\right| \\
& =e^{-\frac{1}{2}\left(\left|z_{+}\right|^{2}+\left|z_{-}\right|^{2}\right)} \sum_{\tilde{n}_{+}, \tilde{n}_{-}=0}^{\infty} \frac{z_{+}^{\tilde{n}_{+}} \bar{z}_{-}^{\tilde{n}_{-}}}{\sqrt{\tilde{n}_{+} ! \tilde{n}_{-} !}}\left|\tilde{n}_{+}\right\rangle\left\langle\tilde{n}_{-}\right| .
\end{aligned}
$$

By taking a state $\mid \psi$ ) on $\mathcal{H}_{q}$, we obtain

$$
\begin{aligned}
\left.\frac{1}{\pi^{2}} \int_{\mathbb{C}^{2}} d^{2} z_{+} d^{2} z_{-} \mid z_{ \pm}\right)\left(z_{ \pm} \mid \psi\right)= & \frac{1}{\pi^{2}} \int_{\mathbb{C}^{2}} d^{2} z_{+} d^{2} z_{-}\left|z_{+}\right\rangle\left\langle z_{-}\right| \\
& \times \sum_{\tilde{n}_{ \pm}=0}^{\infty}\left|\tilde{n}_{-}\right\rangle\left\langle\tilde{n}_{+}\left|\left[\left|z_{+}\right\rangle\left\langle z_{-}\right|\right]^{\ddagger} \psi\right| \tilde{n}_{+}\right\rangle\left\langle\tilde{n}_{-}\right|
\end{aligned}
$$


M. N. Hounkonnou \&S I. Aremua

$$
\begin{aligned}
& =\frac{1}{\pi^{2}} \int_{\mathbb{C}^{2}} d^{2} z_{+} d^{2} z_{-}\left|z_{+}\right\rangle\left\langle z_{-}\right|\left\langle z_{+}|\psi| z_{-}\right\rangle \\
& =\mid \psi),
\end{aligned}
$$

such that

$$
\left.\frac{1}{\pi^{2}} \int_{\mathbb{C}^{2}} d^{2} z_{+} d^{2} z_{-} \mid z_{ \pm}\right)\left(z_{ \pm} \mid \equiv \mathbb{I}_{q}\right.
$$

In order to provide an equivalence between (3.4) and (3.5), let us consider the following relations

$$
\begin{aligned}
\mathbb{I}_{q}|\psi\rangle & =\frac{1}{\pi^{2}} \int_{\mathbb{C}^{2}} d z d \bar{z} d w d \bar{w}|z\rangle\langle w|\langle z|\psi| w\rangle \\
& =\frac{1}{\pi^{2}} \int_{\mathbb{C}^{2}} d z d \bar{z} d u d \bar{u}|z\rangle\langle z+u|\langle z|\psi| z+u\rangle \\
& =\frac{1}{\pi} \int_{\mathbb{C}} d z d \bar{z} \frac{1}{\pi} \int_{\mathbb{C}} d^{2} u e^{-|u|^{2}}|z\rangle\langle z| e^{\bar{u} \overleftarrow{\partial_{\bar{z}}}+u \overrightarrow{\partial_{z}}}\langle z|\psi| z\rangle
\end{aligned}
$$

where $w=z+u$ with $d^{2} w=d^{2} u$, and $e^{u \partial_{z}} f(z)=f(z+u)$. Then, set

$$
\frac{1}{\pi} \int_{\mathbb{C}} d^{2} u e^{-|u|^{2}}|z\rangle\left\langle z\left|e^{\bar{u} \overleftarrow{\partial_{\bar{z}}}+u \overrightarrow{\partial_{z}}}\langle z|\psi| z\rangle=\frac{1}{\pi} \int_{\mathbb{C}} d^{2} u e^{-|u|^{2}}\right| z\right\rangle\langle z| e^{\bar{u} \overleftarrow{\partial_{\bar{z}}}} e^{u \overrightarrow{\partial_{z}}}\langle z|\psi| z\rangle
$$

and

$$
I=|z\rangle\langle z| e^{\bar{u} \overleftarrow{\partial_{\bar{z}}}} e^{u \overrightarrow{\partial_{z}}}\langle z|\psi| z\rangle
$$

We have

$$
\begin{aligned}
I= & {\left[\sum_{n^{\prime}, m^{\prime}=0}^{\infty}\left|n^{\prime}\right\rangle\left\langle m^{\prime}\right| e^{-\bar{z} z} \frac{\bar{z}^{m^{\prime}}}{\sqrt{m^{\prime} !}} \frac{z^{n^{\prime}}}{\sqrt{n^{\prime} !}}\right] e^{\bar{u} \overleftarrow{\partial_{\bar{z}}}} e^{u \overrightarrow{\partial_{z}}}\left[\sum_{n, m=0}^{\infty}\langle m|\psi| n\rangle e^{-\bar{z} z} \frac{\bar{z}^{n}}{\sqrt{n !}} \frac{z^{m}}{\sqrt{m !}}\right] } \\
= & {\left[\sum_{n, m=0}^{\infty} \sum_{n^{\prime}, m^{\prime}=0}^{\infty} \frac{z^{n^{\prime}}}{\sqrt{n^{\prime} !}} \frac{\bar{z}^{n}}{\sqrt{n !}}\left|n^{\prime}\right\rangle\left\langle m^{\prime}\right|\langle m|\psi| n\rangle\right] } \\
& \times\left(e^{-\bar{z} z} \frac{\bar{z}^{m^{\prime}}}{\sqrt{m^{\prime} !}}\right) e^{\bar{u} \overleftarrow{\partial_{\bar{z}}}} e^{u \overrightarrow{\partial_{z}}}\left(e^{-\bar{z} z} \frac{z^{m}}{\sqrt{m !}}\right) .
\end{aligned}
$$

Let

$$
K(z)=\left(e^{-\bar{z} z} \frac{\bar{z}^{m^{\prime}}}{\sqrt{m^{\prime} !}}\right) e^{\bar{u} \overleftarrow{\partial_{\bar{z}}}} e^{u \overrightarrow{\partial_{z}}}\left(e^{-\bar{z} z} \frac{z^{m}}{\sqrt{m !}}\right)
$$

We obtain

$$
K(z)=\frac{1}{\sqrt{m^{\prime} !}} \frac{1}{\sqrt{m !}} \sum_{k=0}^{\infty} \sum_{l=0}^{\infty} \frac{1}{k !}\left(\bar{u}^{k} \partial_{\bar{z}}^{k}\left[\bar{z}^{m^{\prime}} e^{-\bar{z} z}\right]\right) \frac{1}{l !}\left(u^{l} \partial_{z}^{l}\left[z^{m} e^{-\bar{z} z}\right]\right),
$$


which supplies, by performing a radial parametrization,

$$
\begin{aligned}
\frac{1}{\pi} \int_{\mathbb{C}} d^{2} u e^{-|u|^{2}} K(z) \\
=\frac{1}{\sqrt{m^{\prime} !}} \frac{1}{\sqrt{m !}} \sum_{k=0}^{\infty} \sum_{l=0}^{\infty} \frac{1}{\pi} \int_{\mathbb{C}} d^{2} u e^{-|u|^{2}} \frac{\bar{u}^{k}}{k !} \frac{u^{l}}{l !} \partial_{\bar{z}}^{k}\left[\bar{z}^{m^{\prime}} e^{-\bar{z} z}\right] \partial_{z}^{l}\left[z^{m} e^{-\bar{z} z}\right] \\
=\frac{1}{\sqrt{m^{\prime} !}} \frac{1}{\sqrt{m !}} \sum_{k=0}^{\infty} \sum_{l=0}^{\infty} \frac{1}{\pi} \int_{0}^{\infty} r d r e^{-r^{2}} \frac{r^{k+l}}{k ! l !} \int_{0}^{2 \pi} e^{-i(l-k) \phi} d \phi \\
=\frac{1}{\sqrt{m^{\prime} !}} \frac{1}{\sqrt{m !}} \sum_{k=0}^{\infty}\left[\frac{1}{k !} \int_{0}^{m^{\prime}} e^{-\bar{z} z}\right] \partial_{z}^{l}\left[z^{m} e^{-\bar{z} z}\right] \\
=\frac{1}{\sqrt{m^{\prime} !}} \frac{1}{\sqrt{m !}} \sum_{k=0}^{\infty}\left[\frac{1}{k !} \partial_{\bar{z}}^{k}\left[\bar{z}^{m^{\prime}} e^{-\bar{z} z}\right] \partial_{z}^{k}\left[z^{m} e^{-\bar{z} z}\right]\right] .
\end{aligned}
$$

Besides,

$$
\left(e^{-\bar{z} z} \frac{\bar{z}^{m^{\prime}}}{\sqrt{m^{\prime !}}}\right) e^{\overleftarrow{\partial_{\bar{z}}} \overrightarrow{\partial_{z}}}\left(e^{-\bar{z} z} \frac{z^{m}}{\sqrt{m !}}\right)=\frac{1}{\sqrt{m^{\prime} !}} \frac{1}{\sqrt{m !}} \sum_{k=0}^{\infty}\left[\frac{1}{k !} \partial_{\bar{z}}^{k}\left[\bar{z}^{m^{\prime}} e^{-\bar{z} z}\right] \partial_{z}^{k}\left[z^{m} e^{-\bar{z} z}\right]\right]
$$

which implies that

$$
\frac{1}{\pi} \int_{\mathbb{C}} d^{2} u e^{-|u|^{2}} K(z)=\left(e^{-\bar{z} z} \frac{\bar{z}^{m^{\prime}}}{\sqrt{m^{\prime} !}}\right) e^{\overleftarrow{\partial_{\bar{z}}} \overrightarrow{\partial_{z}}}\left(e^{-\bar{z} z} \frac{z^{m}}{\sqrt{m !}}\right)
$$

Then,

$$
\begin{aligned}
& \frac{1}{\pi} \int_{\mathbb{C}} d^{2} u e^{-|u|^{2}}|z\rangle\langle z| e^{\bar{u} \overleftarrow{\partial_{\bar{z}}}+u \overrightarrow{\partial_{z}}}\langle z|\psi| z\rangle \\
& =\left[\sum_{n, m=0}^{\infty} \sum_{n^{\prime}, m^{\prime}=0}^{\infty} \frac{z^{n^{\prime}}}{\sqrt{n^{\prime} !}} \frac{\bar{z}^{n}}{\sqrt{n !}}\left|n^{\prime}\right\rangle\left\langle m^{\prime}\right|\langle m|\psi| n\rangle\right]\left(e^{-\bar{z} z} \frac{\bar{z}^{m^{\prime}}}{\sqrt{m^{\prime} !}}\right) e^{\overleftarrow{\partial_{\bar{z}}} \overrightarrow{\partial_{z}}}\left(e^{-\bar{z} z} \frac{z^{m}}{\sqrt{m !}}\right) \\
& =\left[\sum_{n^{\prime}, m^{\prime}=0}^{\infty}\left|n^{\prime}\right\rangle\left\langle m^{\prime}\right| e^{-\bar{z} z} \frac{\bar{z}^{m^{\prime}}}{\sqrt{m^{\prime} !}} \frac{z^{n^{\prime}}}{\sqrt{n^{\prime} !}}\right] e^{\overleftarrow{\partial_{\bar{z}}} \overrightarrow{\partial_{z}}}\left[\sum_{n, m=0}^{\infty}\langle m|\psi| n\rangle e^{-\bar{z} z} \frac{\bar{z}^{n}}{\sqrt{n !}} \frac{z^{m}}{\sqrt{m !}}\right] \\
& =\mid z) e^{\overleftarrow{\partial_{\bar{z}}} \overrightarrow{\partial_{z}}}(z \mid \psi),
\end{aligned}
$$

allowing to obtain (3.10) under the form:

$$
\begin{aligned}
\mathbb{I}_{q}|\psi\rangle & =\frac{1}{\pi} \int_{\mathbb{C}} d z d \bar{z} \frac{1}{\pi} \int_{\mathbb{C}} d^{2} u e^{-|u|^{2}}|z\rangle\langle z| e^{\bar{u} \overleftarrow{\partial_{\bar{z}}}+u \overrightarrow{\partial_{z}}}\langle z|\psi| z\rangle \\
& \left.=\frac{1}{\pi} \int_{\mathbb{C}} d z d \bar{z} \mid z\right) e^{\overleftarrow{\partial_{\bar{z}}} \overrightarrow{\partial_{z}}}(z \mid \psi),
\end{aligned}
$$

which completes the proof. 
Provided the definition of the upper (or covariant) symbol [20,32] of an appropriate observable $\mathcal{O}$, given by

$$
\left.\mathcal{O}=\frac{1}{\pi^{2}} \int_{\mathbb{C}^{2}} \hat{\mathcal{O}} \mid z_{ \pm}, \tau\right)\left(z_{ \pm}, \tau \mid d^{2} z_{+} d^{2} z_{-}\right.
$$

the upper symbol of the Hamiltonian $H_{q}$ is furnished by the formula

$$
\hat{\mathrm{H}}_{q}\left(z_{ \pm}\right)=\hbar\left(\tilde{\Omega}_{+}\left|z_{+}\right|^{2}+\tilde{\Omega}_{-}\left|z_{-}\right|^{2}-\frac{\tilde{\Omega}}{2}\right)+k_{e, E}
$$

Assume that the term $k_{e, E}=-\frac{1}{2} e\left(E_{1} x_{0}+E_{2} y_{0}\right)$ is a mere constant, and set $H_{q}=$ $\mathcal{H}_{\mathrm{OSC}}+\frac{\tilde{\omega}_{c}}{2} L_{z}+k_{e, E}$ such that $\mathcal{H}_{\mathrm{OSC}}$ and $L_{z}$ are expressed by

$$
\begin{aligned}
\mathcal{H}_{\mathrm{OSC}} & =\frac{1}{2 M}\left(\tilde{p}_{x}-\tilde{p}_{x_{0}}\right)^{2}+\frac{1}{2 M}\left(\tilde{p}_{y}-\tilde{p}_{y_{0}}\right)^{2}+\frac{M \Omega^{2}}{8}\left[\left(x-x_{0}\right)^{2}+\left(y-y_{0}\right)^{2}\right], \\
L_{z} & =\left(x-x_{0}\right)\left(p_{y}-p_{y_{0}}\right)-\left(y-y_{0}\right)\left(p_{x}-p_{x_{0}}\right)
\end{aligned}
$$

with $p_{x_{0}}=-\frac{e B}{2 c} y_{0}, p_{y_{0}}=\frac{e B}{2 c} x_{0}$ and $\tilde{p}_{\nu}^{2}=\left(1-\frac{M \omega_{c}}{2} \theta+\left(\frac{M \Omega}{4} \theta\right)^{2}\right) p_{\nu}^{2}, \nu=x, y$.

Letting now $\Psi(r, \varphi)=R(r) e^{i \rho \varphi}$, where the polar coordinates $(x, y)=(r \sin \varphi, r \cos \varphi)$ with $0<r<\infty$ and $0 \leq \varphi \leq \pi$ are introduced, the stationary Schrödinger equation $\mathcal{H} \Psi=\mathcal{E} \Psi$, where $\mathcal{H}=\mathcal{H}_{\mathrm{OSC}}+\frac{\overline{\tilde{\omega}}_{c}}{2} L_{z}$, is detailed as follows:

$$
\begin{aligned}
& {\left[-\frac{\hbar^{2}}{2 M}\left(1-\frac{M \omega_{c}}{2} \theta+\left(\frac{M \Omega}{4} \theta\right)^{2}\right)\left(\partial_{r}^{2}+\frac{1}{r} \partial_{r}+\frac{1}{r^{2}} \partial_{\varphi}^{2}\right)-i \frac{\hbar}{2} \tilde{\omega}_{c} \partial_{\varphi}+\frac{M \Omega^{2}}{8} r^{2}\right]} \\
& \times \Psi(r, \varphi)=\mathcal{E} \Psi(r, \varphi),
\end{aligned}
$$

engendering the eigenstates and eigenvalues

$$
\Psi_{n, \rho}(r, \varphi)=(-1)^{n} \sqrt{\frac{\xi}{\pi}} \sqrt{\frac{n !}{(n+|\rho|) !}} \exp \left\{-\frac{\xi r^{2}}{2}\right\}(\sqrt{\xi} r)^{|\rho|} L_{n, \theta}^{(|\rho|)}\left(\xi r^{2}\right) e^{i \rho \varphi}
$$

and

$$
\mathcal{E}_{n, \rho, \theta}=\hbar \tilde{\Omega}\left(n+\frac{|\rho|+1}{2}\right)+\frac{\hbar \tilde{\omega}_{c}}{2} \rho-\frac{1}{2} e\left(E_{1} x_{0}+E_{2} y_{0}\right),
$$

respectively. Here, the quantities

$$
L_{n, \theta}^{(|\rho|)}\left(\xi r^{2}\right)=\sum_{m=0}^{n}(-1)^{m}\left(\begin{array}{c}
n+|\rho| \\
n-m
\end{array}\right) \frac{\left(\xi r^{2}\right)^{m}}{m !}
$$

are Laguerre polynomials; $\xi$ is given by

$$
\xi=\sqrt{\frac{(M \Omega / 2 \hbar)^{2}}{1-\frac{M \omega_{c}}{2} \theta+\left(\frac{M \Omega}{4} \theta\right)^{2}}}
$$

and $n=0,1,2, \ldots$ represents the principal quantum number while $\rho=0, \pm 1, \pm 2, \ldots$ stands for the angular moment quantum number. 
The Hamiltonian $H_{q}$, splitting as in (2.28), differs, by the introduction of an additional electric field term, from the model investigated on the Landau diamagnetism and the de Haas-van Alphen effect in various regimes of temperature and magnetic field [28], relevant in condensed matter physics. As a matter of result comparison and in order to highlight the contribution of the introduced electric term, we focus now our study on the thermodynamics of the described system like in [28].

Keeping in mind the resolution of the identity (3.4) satisfied by the family of states $\left.\mid z_{ \pm}, \tau\right)$, we first intend to get for the physical model some inequalities implying the property (3.4) by following the analysis performed in [20]. Then, we derive our thermodynamical quantities. Although some main expressions appear similar in their form to those derived in [20], due to our system parameter redefinition, this investigation has the interesting advantage to offer an easier relation comparison and to point up the contribution of the electric field not considered in previous works.

From (3.26), we derive the thermodynamical potential using the formula [34]:

$$
\Gamma_{\theta}=-\frac{1}{\beta} \sum_{n=0}^{\infty} \sum_{\rho=-\infty}^{\infty} \log \left[1+e^{-\beta\left(\mathcal{E}_{n, \rho, \theta}-\mu\right)}\right]
$$

where $\beta=1 / k_{B} T ; \mu$ is the chemical potential.

The resolution of the identity (3.4) allows to apply the Berezin-Lieb inequalities [18, 20]:

$$
\begin{gathered}
-\frac{1}{\beta \pi^{2}} \int_{\mathbb{C}^{2}} \log \left(1+e^{-\beta\left(\hat{\mathrm{H}}_{q}-\mu\right)}\right) d^{2} z_{+} d^{2} z_{-} \leq \Gamma_{\theta}, \\
\Gamma_{\theta} \leq-\frac{1}{\beta \pi^{2}} \int_{\mathbb{C}^{2}} \log \left(1+e^{-\beta\left(\check{\mathrm{H}}_{q}-\mu\right)}\right) d^{2} z_{+} d^{2} z_{-} .
\end{gathered}
$$

By using the lower and upper symbols of the Hamiltonian $H_{q}$, and performing the angular integrations, where $u_{+}=\left|z_{+}\right|^{2}, v_{-}=\left|z_{-}\right|^{2}$ with $z_{+}=r e^{i \varphi}, z_{-}=\rho e^{i \phi}, r, \rho \geq 0$, $\varphi, \phi \in[0,2 \pi)$, we get

$$
\begin{gathered}
-\frac{1}{\beta} \int_{0}^{\infty} d u_{+} \int_{0}^{\infty} d v_{-} \log \left(1+e^{-\beta\left(\hbar\left(\tilde{\Omega}_{+} u_{+}+\tilde{\Omega}_{-} v_{-}-\frac{\tilde{\Omega}}{2}\right)-\mu_{e, E}\right)}\right) \leq \Gamma_{\theta}, \\
\Gamma_{\theta} \leq-\frac{1}{\beta} \int_{0}^{\infty} d u_{+} \int_{0}^{\infty} d v_{-} \log \left(1+e^{-\beta\left(\hbar\left(\tilde{\Omega}_{+} u_{+}+\tilde{\Omega}_{-} v_{-}+\frac{\tilde{\Omega}}{2}\right)-\mu_{e, E}\right)}\right),
\end{gathered}
$$

where $\mu_{e, E}=\mu+\frac{1}{2} e\left(E_{1} x_{0}+E_{2} y_{0}\right)$.

Setting $u=\beta \hbar\left(\tilde{\Omega}_{+} u_{+}+\tilde{\Omega}_{-} v_{-}\right), v=\beta \hbar \tilde{\Omega}_{+} u_{+}$, performing an integration by parts and introducing the control parameters $\tilde{\kappa}_{ \pm}^{\prime}=\exp \left(\beta\left(\mu_{e, E} \pm \frac{\hbar \tilde{\Omega}}{2}\right)\right)=\exp (-\beta k) \cdot \tilde{\kappa}_{ \pm}$, where $\tilde{\kappa}_{ \pm}=$ $\exp \left(\beta\left(\mu \pm \frac{\hbar \tilde{\Omega}}{2}\right)\right),(3.31)$ is reduced to

$$
\phi\left(\tilde{\kappa}_{+}^{\prime}\right) \leq \Gamma_{\theta} \leq \phi\left(\tilde{\kappa}_{-}^{\prime}\right)
$$


where $\phi\left(\tilde{\kappa}^{\prime}\right)$ takes the form

$$
\begin{aligned}
\phi\left(\tilde{\kappa}^{\prime}\right) & =-\frac{\tilde{\kappa}^{\prime}}{2 \beta\left(\beta \hbar \omega_{0}\right)^{2}} \int_{0}^{\infty} \frac{u^{2} e^{-u}}{1+\tilde{\kappa}^{\prime} e^{-u}} d u \\
& = \begin{cases}\frac{1}{\beta\left(\beta \hbar \omega_{0}\right)^{2}} F_{3}\left(-\tilde{\kappa}^{\prime}\right) & \text { for } \tilde{\kappa}^{\prime} \leq 1, \\
\frac{1}{\beta\left(\beta \hbar \omega_{0}\right)^{2}}\left[-\frac{\left(\log \tilde{\kappa}^{\prime}\right)^{3}}{6}-\frac{\pi^{2} \log \tilde{\kappa}^{\prime}}{6}+F_{3}\left(-\tilde{\kappa}^{\prime-1}\right)\right] & \text { for } \tilde{\kappa}^{\prime}>1 .\end{cases}
\end{aligned}
$$

The function $F_{s}$ is of the Riemann-Fermi-Dirac type [20]:

$$
F_{s}(z)=\sum_{n=1}^{\infty} \frac{z^{n}}{n^{s}}
$$

In the high-temperature limit case, the assumption $\left|\mu_{e, E} \pm \frac{\hbar \tilde{\Omega}}{2}\right| \gg \beta$ gives $\tilde{\kappa}_{ \pm}^{\prime} \approx 1$ so that using (3.31) and (3.32) the thermodynamical potential $\Gamma_{\theta}$ can be approximated by

$$
\Gamma_{\theta} \approx \frac{1}{\beta^{3} \hbar^{2}} \frac{F_{3}(-1)}{\omega_{0}^{2}} \approx-0.901543 \times \frac{1}{\beta}\left(\frac{1}{\beta \hbar \omega_{0}}\right)^{2} .
$$

Consider the expression of the function $\phi$ as follows [20]:

$$
\phi\left(\tilde{\kappa}_{ \pm}^{\prime}\right)=A \mp \frac{\Delta}{2}+S_{ \pm},
$$

where in our considered physical model situation

$$
\begin{aligned}
A & =-\frac{\mu_{e, E}}{2}\left[\frac{1}{3}\left(\frac{\mu_{e, E}}{\hbar \omega_{0}}\right)^{2}+\frac{1}{4}\left(\frac{\tilde{\Omega}}{\omega_{0}}\right)^{2}+\frac{\pi^{2}}{3}\left(\frac{1}{\beta \hbar \omega_{0}}\right)^{2}\right], \\
\frac{\Delta}{2} & =\frac{\hbar \tilde{\Omega}}{2}\left[\frac{1}{2}\left(\frac{\mu_{e, E}}{\hbar \omega_{0}}\right)^{2}+\frac{1}{24}\left(\frac{\tilde{\Omega}}{\omega_{0}}\right)^{2}+\frac{\pi^{2}}{6}\left(\frac{1}{\beta \hbar \omega_{0}}\right)^{2}\right], \\
S_{ \pm} & =\frac{1}{\beta\left(\beta \hbar \omega_{0}\right)^{2}} F_{3}\left(-e^{-\beta\left(\mu_{e, E} \pm \frac{\hbar \tilde{\Omega}}{2}\right)}\right) .
\end{aligned}
$$

At low temperature, $S_{ \pm}$can be approximated by

$$
S_{0}=\frac{1}{\beta\left(\beta \hbar \omega_{0}\right)^{2}} F_{3}\left(-e^{-\beta \mu_{e, E}}\right) .
$$

Considering the following ratio

$$
\frac{\Delta}{\left|A+S_{0}\right|}=\frac{\hbar \tilde{\Omega}}{\mu_{e, E}}\left[\frac{3+\pi^{2}\left(\frac{1}{\beta \mu_{e, E}}\right)^{2}+\frac{1}{4}\left(\frac{\hbar \tilde{\Omega}}{\mu_{e, E}}\right)^{2}}{1+\pi^{2}\left(\frac{1}{\beta \mu_{e, E}}\right)^{2}+\frac{3}{4}\left(\frac{\hbar \tilde{\Omega}}{\mu_{e, E}}\right)^{2}-\left(\frac{1}{\beta \mu_{e, E}}\right)^{3} F_{3}\left(-e^{-\beta \mu_{e, E}}\right)}\right]
$$


which tends to zero at low temperature, namely $\mu_{e, E} \gg \hbar \tilde{\Omega} / 2$ and $\mu_{e, E} \gg 1 / \beta$, the thermodynamical potential can be obtained as

$$
\begin{aligned}
\Gamma_{\theta} \approx & A+S_{0} \\
= & -\frac{\mu_{e, E}}{2}\left[\frac{1}{3}\left(\frac{\mu_{e, E}}{\hbar \omega_{0}}\right)^{2}+\frac{1}{4}\left(\frac{\tilde{\Omega}}{\omega_{0}}\right)^{2}+\frac{\pi^{2}}{3}\left(\frac{1}{\beta \hbar \omega_{0}}\right)^{2}\right. \\
& \left.-\frac{2}{\beta \mu_{e, E}\left(\beta \hbar \omega_{0}\right)^{2}} F_{3}\left(-e^{-\beta \mu_{e, E}}\right)\right] .
\end{aligned}
$$

The average number of electrons is given by

$$
\begin{aligned}
\left\langle N_{e}\right\rangle & \approx-\partial_{\mu}\left(A+S_{0}\right) \\
& =\left(\frac{\mu_{e, E}}{\hbar \omega_{0}}\right)^{2}\left[\frac{1}{2}+\frac{1}{8}\left(\frac{\hbar \tilde{\Omega}}{\mu_{e, E}}\right)^{2}+\frac{\pi^{2}}{6}\left(\frac{1}{\beta \mu_{e, E}}\right)^{2}+\left(\frac{1}{\beta \mu_{e, E}}\right)^{2} F_{2}\left(-e^{-\beta \mu_{e, E}}\right)\right] \\
& \approx \frac{1}{2}\left(\frac{\mu_{e, E}}{\hbar \omega_{0}}\right)^{2} \text { for } \mu_{e, E} \gg \hbar \tilde{\Omega} / 2 \text { and } \mu_{e, E} \gg 1 / \beta
\end{aligned}
$$

The magnetic moment $\mathcal{M}_{\theta}=-\left(\frac{\partial \Gamma_{\theta}}{\partial B}\right)_{\mu}$ is derived as follows

$$
\begin{aligned}
\mathcal{M}_{\theta}= & \frac{e \mu}{4 M c}\left[\frac{\omega_{c}}{\omega_{0}^{2}}-\frac{M \theta}{4 \omega_{0}^{2}}\left(2 \omega_{c}^{2}+\Omega^{2}\right)+2 \frac{\omega_{c}}{\omega_{0}^{2}}\left(\frac{M \Omega \theta}{4}\right)^{2}\right] \\
& +\frac{e^{2}}{8 M c}\left(E_{1} x_{0}+E_{2} y_{0}\right)\left[\frac{\omega_{c}}{\omega_{0}^{2}}-\frac{M \theta}{4 \omega_{0}^{2}}\left(2 \omega_{c}^{2}+\Omega^{2}\right)+2 \frac{\omega_{c}}{\omega_{0}^{2}}\left(\frac{M \Omega \theta}{4}\right)^{2}\right]
\end{aligned}
$$

which provides the susceptibility $\chi_{\theta}=\frac{\partial \mathcal{M}_{\theta}}{\partial B}$ obtained in the following form

$$
\begin{aligned}
\chi_{\theta}= & \left(\frac{e}{2 M c \omega_{0}}\right)^{2} \mu\left[1-\frac{3}{2} M \theta \omega_{c}-\left(M \theta \omega_{0}\right)^{2}+6\left(\frac{M \Omega \theta}{4}\right)^{2}\right] \\
& +\frac{e^{3}}{8\left(M c \omega_{0}\right)^{2}}\left(E_{1} x_{0}+E_{2} y_{0}\right)\left[1-\frac{3}{2} M \theta \omega_{c}-\left(M \theta \omega_{0}\right)^{2}+6\left(\frac{M \Omega \theta}{4}\right)^{2}\right] .
\end{aligned}
$$

In both expressions (3.44) and (3.45), the second terms stand for the contributions induced by the presence of the electric field. The highest the electric potential is at the reference point of position $\overrightarrow{R_{0}}=\left(x_{0}, y_{0}\right)$, the highest is the electric field contribution.

Remark 3.1. In the absence of the electric field, this model is reduced to that described by the Fock-Darwin Hamiltonian investigated in the study of the orbital magnetism of a two-dimensional noncommutative confined system [32]. In that work, it has been shown that the degeneracy of Landau levels can be lifted via the $\theta$-term at weak magnetic field limit, i.e. for $\omega_{c} \ll \omega_{0}$. 
By use of the Poisson summation formula [34] in the sum over $n$ and $\rho$ in (3.29), we obtain

$$
\Gamma_{\theta}=\Gamma_{\theta}^{0}+\Gamma_{\theta}^{L}+\Gamma_{\theta}^{\mathrm{OSC}}
$$

where

$$
\begin{aligned}
\Gamma_{\theta}^{0}= & -\frac{1}{\beta\left(\hbar \omega_{0}\right)^{2}} \int_{0}^{\infty} d \varepsilon \int_{0}^{\infty} d \eta \log \left(1+e^{-\beta\left(\varepsilon+\eta-\mu_{e, E}\right)}\right)+\frac{1}{12} \mu_{e, E} \\
\Gamma_{\theta}^{L}= & \frac{\mu_{e, E}}{24}\left(\frac{\omega_{c}-\Theta_{M, \omega_{c}, \theta}}{\omega_{0}}\right)^{2}, \\
\Gamma_{\theta}^{\mathrm{OSC}}= & \frac{1}{2 \pi \beta} \sum_{k=1}^{\infty}(-1)^{k}\left[\left(\frac{\tilde{\Omega}}{\omega_{0}}\right)^{2} \frac{1}{k^{2}}-\frac{\pi^{2}}{3}\right] \times \frac{\sin \left\{2 \pi_{\tilde{\Omega}} k \mu_{e, E}\right\}}{\operatorname{Sinh}_{k, \tilde{\Omega}}} \\
& +\frac{1}{2 \pi \beta} \sum_{\sigma= \pm} \sum_{l=1}^{\infty} \frac{\tilde{\Omega}_{\sigma}}{\tilde{\Omega}} \frac{1}{l^{2}} \frac{\sin \left\{2 \pi_{\tilde{\Omega}_{\sigma}} l \mu_{e, E}\right\}}{\operatorname{Sinh}_{l, \tilde{\Omega}_{\sigma}}}+\frac{1}{\pi \beta} \sum_{\sigma= \pm} \sum_{k=1}^{\infty} \sum_{l=1}^{\infty} \frac{(-1)^{k}}{l} \\
& \times\left[\frac{\sin \left\{\pi_{\tilde{\Omega}} \mu_{e, E} K_{k, l, \tilde{\Omega}, \sigma ;-}\right\} \cos \left\{\pi_{\tilde{\Omega}} \mu_{e, E} K_{k, l, \tilde{\Omega}, \sigma ;+}\right\}}{K_{k, l, \tilde{\Omega}, \sigma ;-} \operatorname{Sinh}_{l, \tilde{\Omega}_{\sigma}}}\right. \\
& \left.+\frac{\sin \left\{\pi_{\tilde{\Omega}} \mu_{e, E} K_{k, l, \tilde{\Omega}, \sigma ;+}\right\} \cos \left\{\pi_{\tilde{\Omega}} \mu_{e, E} K_{k, l, \tilde{\Omega}, \sigma ;-}\right\}}{K_{k, l, \tilde{\Omega}, \sigma ;+} \operatorname{Sinh}_{l, \tilde{\Omega}_{\sigma}}}\right]
\end{aligned}
$$

with the relation $\mu \gg T$ assumed.

From the thermodynamical potential $\Gamma_{\theta}$, the magnetic moment $\mathcal{M}_{\theta}=-\left(\frac{\partial \Gamma_{\theta}}{\partial B}\right)_{\mu}$ can be derived to give the following contributions:

$$
\mathcal{M}_{\theta}=\mathcal{M}_{\theta}^{L}+\mathcal{M}_{\theta, 1}^{\mathrm{OSC}}+\mathcal{M}_{\theta, 2}^{\mathrm{OSC}}+\mathcal{M}_{\theta, 3}^{\mathrm{OSC}},
$$

where

$$
\begin{aligned}
& \mathcal{M}_{\theta}^{0}=-\left(\frac{\partial \Gamma_{\theta}^{0}}{\partial B}\right)_{\mu}=0 \\
& \mathcal{M}_{\theta}^{L}=-\left(\frac{\partial \Gamma_{\theta}^{L}}{\partial B}\right)_{\mu}=-\frac{\mu_{e, E}}{12}\left[\frac{\omega_{c}-\Theta_{M, \omega_{c}, \theta}}{\omega_{0}^{2}}\right] \mathcal{I}_{e, B, \theta, M}, \\
& \mathcal{M}_{\theta, 1}^{\mathrm{OSC}}=-\frac{1}{2 \pi \beta} \sum_{k=1}^{\infty}(-1)^{k}\left[\frac{1}{k^{2} \omega_{0}^{2}}\left(\frac{2 e \omega_{c}}{M c}\left(\frac{\tilde{\Omega}}{\Omega}\right)^{2}+\Omega^{2} B_{\theta}\right)\right. \\
& \times \frac{\sin \left\{2 \pi_{\tilde{\Omega}} k \mu_{e, E}\right\}}{\operatorname{Sinh}_{k, \tilde{\Omega}}}-\frac{2 \pi k}{\hbar \operatorname{Sinh}_{k, \tilde{\Omega}}}\left[\left(\frac{\tilde{\Omega}}{\omega_{0}}\right)^{2} \frac{1}{k^{2}}-\frac{\pi^{2}}{3}\right] \frac{\mathcal{K}_{\omega_{c}, e, \theta, B, M}}{\tilde{\Omega}^{2}} \\
& \left.\times\left[\mu_{e, E} \cos \left\{2 \pi_{\tilde{\Omega}} k \mu_{e, E}\right\}-\frac{\pi}{\beta} \operatorname{Coth}_{k, \tilde{\Omega}} \times \sin \left\{2 \pi_{\tilde{\Omega}} k \mu_{e, E}\right\}\right]\right],
\end{aligned}
$$




$$
\begin{aligned}
& \mathcal{M}_{\theta, 2}^{\mathrm{OSC}}=-\frac{1}{2 \pi \beta} \sum_{\sigma= \pm} \sum_{l=1}^{\infty} \frac{1}{l^{2}} \\
& \times\left[\left[ \pm \frac{1}{\tilde{\Omega}^{2}}\left[\mathcal{L}_{e, B, \theta, M}-\frac{\tilde{\omega}_{c}}{2} \mathcal{K}_{\omega_{c}, e, \theta, B, M}\right]\right] \times \frac{\sin \left\{2 \pi_{\tilde{\Omega}_{\sigma}} l \mu_{e, E}\right\}}{\operatorname{Sinh}_{l, \tilde{\Omega}_{\sigma}}}\right. \\
& -\frac{\tilde{\Omega}_{\sigma}}{\tilde{\Omega}} \frac{2 \pi l}{\hbar \operatorname{Sinh}_{l, \tilde{\Omega}_{\sigma}}} \times \frac{1}{2 \tilde{\Omega}_{\sigma}^{2}}\left[\mathcal{K}_{\omega_{c}, e, \theta, B, M} \pm \mathcal{I}_{e, B, \theta, M}\right] \\
& \times\left[\mu_{e, E} \cos \left\{2 \pi_{\tilde{\Omega}_{\sigma}} l \mu_{e, E}\right\}-\frac{\pi}{\beta} \operatorname{Coth}_{l, \tilde{\Omega}_{\sigma}} \times \sin \left\{2 \pi_{\tilde{\Omega}_{\sigma}} l \mu_{e, E}\right\}\right],
\end{aligned}
$$

$$
\begin{aligned}
& \mathcal{M}_{\theta, 3}^{\mathrm{OSC}}=-\frac{1}{\pi \beta} \sum_{\sigma= \pm} \sum_{k=1}^{\infty} \sum_{l=1}^{\infty} \frac{(-1)^{k}}{l} \\
& \times\left[\left[\frac{1}{\left[K_{k, l, \tilde{\Omega}, \sigma ;-} \operatorname{Sinh}_{l, \tilde{\Omega}_{\sigma}}\right]} \times \pi_{\tilde{\Omega}} \mu_{e, E}\right.\right. \\
& \times\left[\left[k \cos \left\{2 \pi_{\tilde{\Omega}} k\right\}-l \frac{\tilde{\Omega}}{\tilde{\Omega}_{\sigma}} \cos \left\{2 \pi_{\tilde{\Omega}_{\sigma}} l \mu_{e, E}\right\}\right] \times\left[-\frac{\mathcal{K}_{\omega_{c}, e, \theta, B, M}}{\tilde{\Omega}}\right]\right. \\
& \left. \pm l \cos \left\{2 \pi_{\tilde{\Omega}_{\sigma}} l \mu_{e, E}\right\} \times \frac{1}{\tilde{\Omega}_{\sigma}^{2}}\left(\mathcal{L}_{\omega_{c}, e, \theta, B, M}-\frac{\tilde{\omega}_{c}}{2} \mathcal{K}_{\omega_{c}, e, \theta, B, M}\right)\right] \\
& +\frac{1}{\left[K_{k, l, \tilde{\Omega}, \sigma ;+} \operatorname{Sinh}_{l, \tilde{\Omega}_{\sigma}}\right]} \times \pi_{\tilde{\Omega}} \mu_{e, E} \\
& \times\left[\left[k \cos \left\{2 \pi_{\tilde{\Omega}} k\right\}-l \frac{\tilde{\Omega}}{\tilde{\Omega}_{\sigma}} \cos \left\{2 \pi_{\tilde{\Omega}_{\sigma}} l \mu_{e, E}\right\}\right] \times\left[-\frac{\mathcal{K}_{\omega_{c}, e, \theta, B, M}}{\tilde{\Omega}}\right]\right. \\
& \left.\mp l \cos \left\{2 \pi_{\tilde{\Omega}_{\sigma}} l \mu_{e, E}\right\} \times \frac{1}{\tilde{\Omega}_{\sigma}^{2}}\left(\mathcal{L}_{\omega_{c}, e, \theta, B, M}-\frac{\tilde{\omega}_{c}}{2} \mathcal{K}_{\omega_{c}, e, \theta, B, M}\right)\right] \\
& -\frac{\sin \left\{\pi_{\tilde{\Omega}} \mu_{e, E} K_{k, l, \tilde{\Omega}, \sigma ;+}\right\} \cos \left\{\pi_{\tilde{\Omega}} \mu_{e, E} K_{k, l, \tilde{\Omega}, \sigma ;-}\right\}}{\left[K_{k, l, \tilde{\Omega}, \sigma ;+} \operatorname{Sinh}_{l, \tilde{\Omega}_{\sigma}}\right]^{2}} \\
& \times\left[\mp \frac{l}{\left(\tilde{\Omega}_{\sigma}\right)^{2}}\left(\mathcal{L}_{e, B, \theta, M}-\frac{\tilde{\omega}_{c}}{2} \mathcal{K}_{\omega_{c}, e, \theta, B, M}\right) \operatorname{Sinh}_{l, \tilde{\Omega}_{\sigma}}\right. \\
& \left.-K_{k, l, \tilde{\Omega}, \sigma ;+} \frac{2 \pi^{2} l}{\beta \hbar} \operatorname{Cosh}_{l, \tilde{\Omega}_{\sigma}} \times \frac{1}{2\left(\tilde{\Omega}_{\sigma}\right)^{2}}\left(\mathcal{K}_{\omega_{c}, e, \theta, B, M} \pm \mathcal{I}_{e, B, \theta, M}\right)\right] \\
& -\frac{\sin \left\{\pi_{\tilde{\Omega}} \mu_{e, E} K_{k, l, \tilde{\Omega}, \sigma ;-}\right\} \cos \left\{\pi_{\tilde{\Omega}} \mu_{e, E} K_{k, l, \tilde{\Omega}, \sigma ;+}\right\}}{\left[K_{k, l, \tilde{\Omega}, \sigma ;-} \operatorname{Sinh}_{l, \tilde{\Omega}_{\sigma}}\right]^{2}}
\end{aligned}
$$




$$
\begin{aligned}
& \times\left[ \pm \frac{l}{\left(\tilde{\Omega}_{\sigma}\right)^{2}}\left(\mathcal{L}_{e, B, \theta, M}-\frac{\tilde{\omega}_{c}}{2} \mathcal{K}_{\omega_{c}, e, \theta, B, M}\right) \operatorname{Sinh}_{l, \tilde{\Omega}_{\sigma}}-K_{k, l, \tilde{\Omega}, \sigma ;-}\right. \\
& \left.\left.\left.\times \frac{2 \pi^{2} l}{\beta \hbar} \operatorname{Cosh}_{l, \tilde{\Omega}_{\sigma}} \times \frac{1}{2\left(\tilde{\Omega}_{\sigma}\right)^{2}}\left(\mathcal{K}_{\omega_{c}, e, \theta, B, M} \pm \mathcal{I}_{e, B, \theta, M}\right)\right]\right]\right]
\end{aligned}
$$

with

$$
\begin{aligned}
\Theta_{M, \omega_{c}, \theta} & =\frac{M \omega_{c}^{2} \theta}{4}+M \omega_{0}^{2} \theta, \quad B_{\theta}=\frac{e \theta}{2 c}\left(\frac{e B}{4 c} \theta-1\right), \\
\pi_{\tilde{\Omega}} & =\frac{\pi}{\hbar \tilde{\Omega}}, \quad \pi_{\tilde{\Omega}_{\sigma}}=\frac{\pi}{\hbar \tilde{\Omega}_{\sigma}}, \quad k \pm \frac{\tilde{\Omega}}{\tilde{\Omega}_{\sigma}} l=K_{k, l, \tilde{\Omega}, \sigma ; \pm}, \\
\sinh \left\{\frac{2 \pi^{2} l}{\beta \hbar \tilde{\Omega}_{\sigma}}\right\} & =\operatorname{Sinh}_{l, \tilde{\Omega}_{\sigma}}, \quad \sinh \left\{\frac{2 \pi^{2} k}{\beta \hbar \tilde{\Omega}}\right\}=\operatorname{Sinh}_{k, \tilde{\Omega}}, \\
\cosh \left\{\frac{2 \pi^{2} l}{\beta \hbar \tilde{\Omega}_{\sigma}}\right\} & =\operatorname{Cosh}_{l, \tilde{\Omega}_{\sigma}}, \quad \cosh \left\{\frac{2 \pi^{2} k}{\beta \hbar \tilde{\Omega}}\right\}=\operatorname{Cosh}_{k, \tilde{\Omega}}, \\
\mathcal{I}_{e, B, \theta, M} & =\frac{e}{M c}\left(1-\frac{e B}{2 c} \theta\right), \\
\mathcal{K}_{\omega_{c}, e, \theta, B, M} & =\frac{\omega_{c} e \tilde{\Omega}}{M c \Omega^{2}}+\frac{\Omega^{2}}{\tilde{\Omega}} \frac{e \theta}{4 c}\left(\frac{e B}{4 c} \theta-1\right), \\
\mathcal{L}_{e, B, \theta, M} & =\frac{e \tilde{\Omega}}{2 M c}\left(1-\frac{e B}{2 c} \theta\right) .
\end{aligned}
$$

Remark 3.2. In the situation where the $\theta$ - parameter is switched off, we obtain:

$$
\begin{aligned}
\Theta_{M, \omega_{c} \theta=0} & =0, \quad B_{\theta=0}=0, \\
\mathcal{I}_{e, B, \theta=0, M} & =\frac{e}{M c}, \quad \mathcal{K}_{\omega_{c}, e, \theta=0, B, M}=\frac{e \omega_{c}}{M c \Omega}, \quad \mathcal{L}_{e, B, \theta=0, M}=\frac{e \Omega}{2 M c},
\end{aligned}
$$

where we get $\tilde{\Omega} \equiv \Omega, \tilde{\omega}_{c} \equiv \omega_{c}$ and $\tilde{\Omega}_{\sigma} \equiv \Omega_{\sigma}=\frac{\Omega \pm \omega_{c}}{2}$. The term $k_{e, E}=-\frac{1}{2} e\left(E_{1} x_{0}+\right.$ $\left.E_{2} y_{0}\right)$ giving $\mu_{e, E}$ contributes to the chemical potential $\mu$. The expressions (3.47)-(3.49) are identified for $k_{e, E}=0$ with those derived in [28] for the Hamiltonian describing a two-dimensional electrons confined by an isotropic harmonic potential in a perpendicular magnetic field, linked to the Landau problem in the commutative case, for which CS have been constructed on the Fock Hilbert space [20]. Besides, the analysis done in [51] for an ideal fermion gas may also permit to understand the behavior of the studied physical system.

\section{Matrix Vector Coherent States for $\mathbb{H}^{\operatorname{dim}}$}

\subsection{General VCS construction formalism}

As a matter of clarity, let us briefly recall here the synoptic scheme of VCS construction as developed in [2], which will be illustrated in the sequel for the case of noncommutative space. Let $X$ denotes the parameter space for defining the VCS equipped with a topology. $X$ is usually chosen as a locally compact space equipped with a measure $\mu$. Let $\mathfrak{H}$ and $\mathfrak{K}$ be two complex separable Hilbert spaces of infinite or finite dimensions $(1+\operatorname{dim}(\mathfrak{H}))$ and $\operatorname{dim}(\mathfrak{K})$, 
respectively. In $\mathfrak{H}$ and $\mathfrak{K}$, consider specific orthonormal bases $\left\{\phi_{k}\right\}_{k=0}^{\operatorname{dim} \mathfrak{H}}$ and $\left\{\chi^{i}\right\}_{i=1}^{\operatorname{dim} \mathfrak{K}}$. Let $B_{2}(\mathfrak{K})$ be the space of Hilbert-Schmidt operators on $\mathfrak{K}$ which is a Hilbert space under the scalar product $\langle A, B\rangle=\operatorname{Tr}\left[A^{*} B\right]$, for $A, B \in B_{2}(\mathfrak{K})$. Furthermore, let $F_{k}: X \rightarrow B_{2}(\mathfrak{K}), k=$ $1,2, \ldots, \operatorname{dim}(\mathfrak{K})$, be a set of continuous mappings satisfying the conditions:

(i) for each $x \in X, 0<\mathcal{N}(x):=\sum_{k=0}^{\operatorname{dim}(\mathfrak{H})} \operatorname{Tr}\left[\left|F_{k}(x)\right|^{2}\right]<\infty$, where $\left|F_{k}(x)\right|=$ $\left[F_{k}(x) F_{k}(x)^{*}\right]^{\frac{1}{2}}$ denotes the positive part of the operator $F_{k}(x)$. Tr denotes the trace given by

$$
\operatorname{Tr}\left[\left|F_{k}(x)\right|^{2}\right]=\sum_{i=1}^{\operatorname{dim}(\mathfrak{K})}\left\langle\chi^{i} \mid\left(\left|F_{k}(x)\right|\right)^{2} \chi^{i}\right\rangle
$$

(ii) for each $x \in X$, there exists a bounded linear map $T(x): \mathfrak{K} \rightarrow \mathfrak{K} \otimes \mathfrak{H}$, defined by

$$
T(x) \chi=(\mathcal{N}(x))^{-\frac{1}{2}} \sum_{k=0}^{\operatorname{dim}(\mathfrak{H})} F_{k}(x) \chi \otimes \phi_{k}, \quad \chi \in \mathfrak{K} ;
$$

(iii) $I_{\mathfrak{K}}$, the identity operator on $\mathfrak{K}$, admits the representation (in the weak sense)

$$
\int_{X} d \mu(x) F_{k}(x) F_{l}(x)^{*}=\delta_{k l} I_{\mathfrak{K}}, \quad k, l=0,1,2, \ldots, \operatorname{dim}(\mathfrak{H}) .
$$

VCS $|x ; \chi\rangle \in \mathfrak{K} \otimes \mathfrak{H}$ are defined for each $x \in X$ and $\chi \in \mathfrak{K}$, in a general way, by the relation

$$
|x ; X\rangle=T(x) \chi=(\mathcal{N}(x))^{-\frac{1}{2}} \sum_{k=0}^{\operatorname{dim}(\mathfrak{H})} F_{k}(x) \chi \otimes \phi_{k},
$$

from which one obtains, for the particular case of the basis $\left\{\chi^{i}\right\}_{i=1}^{\operatorname{dim} \mathfrak{K}}$

$$
|x ; i\rangle:=\left|x ; \chi^{i}\right\rangle, \quad i=1,2, \ldots, \operatorname{dim}(\mathfrak{K}) .
$$

Let us mention that the VCS $|x ; i\rangle$ may not be linearly independent and sometimes may cancel. $|x ; \chi\rangle$ can be always written as a linear combination as follows:

$$
|x ; \chi\rangle=\sum_{i=1}^{\operatorname{dim}(\mathfrak{K})} c_{i}|x ; i\rangle, \quad \text { where } \quad \chi=\sum_{i=1}^{\operatorname{dim}(\mathfrak{K})} c_{i} \chi^{i}, \quad c_{i} \in \mathbb{C} .
$$

The properties of the family $\{|x ; i\rangle\}_{i=1}^{\operatorname{dim} \mathfrak{K}}$ are evidenced in the following result.

Proposition 4.1. The VCS $|x ; i\rangle$ satisfy

(a) the normalization condition

$$
\sum_{i=1}^{\operatorname{dim}(\mathfrak{K})}|\| x ; i\rangle \|^{2}=1
$$


(b) the resolution of the identity

$$
\sum_{i=1}^{\operatorname{dim}(\mathfrak{K})} \int_{X} d \mu(x) \mathcal{N}(x)|x ; i\rangle\langle x ; i|=I_{\mathfrak{K}} \otimes I_{\mathfrak{H}}
$$

the sum and integral converging in the weak sense.

Proof. See [2].

\subsection{VCS construction in a noncommutative Hilbert space}

Let $\mathcal{M}_{4}(\mathbb{C})$, the space of $4 \times 4$ complex matrices, be a locally compact space equipped with a measure $d \mu$ as the parameter space defining the VCS. Consider the quantum Hilbert space $\mathcal{H}_{q}$ of Hilbert-Schmidt operators acting on the noncommutative configuration (Hilbert) space $\mathcal{H}_{c}=\operatorname{span}\{|n\rangle, n \in \mathbb{N}\}$. Let $F_{n}(\mathfrak{Z}): \mathcal{M}_{4}(\mathbb{C}) \rightarrow \mathcal{B}\left(\mathcal{H}_{c}\right)$, where $\mathcal{B}\left(\mathcal{H}_{c}\right)$ is the set of bounded operators on $\mathcal{H}_{c}$, be a set of continuous mappings satisfying the conditions:

(i) for each $\mathfrak{Z} \in \mathcal{M}_{4}(\mathbb{C})$, the following normalization condition

$$
0<\mathcal{N}(\mathfrak{Z})=\sum_{n \in \mathbb{N}} \operatorname{tr}_{c}\left[\left|F_{n}(\mathfrak{Z})\right|^{2}\right]<\infty
$$

is satisfied, where $\operatorname{tr}_{c}$ stands for the trace over $\mathcal{H}_{c}$ and $\left|F_{n}(\mathfrak{Z})\right|^{2}=\left[F_{n}(\mathfrak{Z}) F_{n}(\mathfrak{Z})^{*}\right]^{1 / 2}$ denotes the positive part of the operator $F_{n}(\mathfrak{Z})$;

(ii) for each $\mathfrak{Z} \in \mathcal{M}_{4}(\mathbb{C})$, there exists a bounded linear map $T(\mathfrak{Z}): \mathbb{C}^{4} \rightarrow \mathbb{C}^{4} \otimes \mathcal{H}_{c}$ defined by

$$
T(\mathfrak{Z}) \chi^{j}=(\mathcal{N}(\mathfrak{Z}))^{-1 / 2} \sum_{n \in \mathbb{N}} F_{n}(\mathfrak{Z})\left|\chi^{j}, n\right\rangle, \quad j=1,2,3,4
$$

(iii) for each $\mathfrak{Z} \in \mathcal{M}_{4}(\mathbb{C})$, the following expression given on $\mathbb{C}^{4} \otimes \mathcal{H}_{c}$ holds:

$$
F_{n}(\mathfrak{Z})\left|\chi^{j}, \tilde{n}\right\rangle=\frac{\mathfrak{Z}^{n} \overline{\mathfrak{Z}}^{\tilde{n}}}{\sqrt{R(n) R(\tilde{n})}}\left|\chi^{j}, \tilde{n}\right\rangle,
$$

where $\mathfrak{Z}=\operatorname{diag}\left(z_{1}, z_{2}, z_{3}, z_{4}\right), z_{j}=r_{j} e^{i \theta_{j}}$ with $r_{j} \geq 0, \theta_{j} \in[0,2 \pi)$ and $R(n)=n ! \mathbb{I}_{4}$.

With this setup and following the construction provided above, based on [2], and taking into account $[23,55]$, the set of vectors formally given by

$$
\begin{aligned}
\mid \mathfrak{Z}, \mathfrak{W}, \tau, j, \tilde{n}, \tilde{m})= & (\mathcal{N}(\mathfrak{Z}, \mathfrak{W}))^{-1 / 2} \sum_{n, m=0}^{\infty} \frac{\mathfrak{Z}^{n} \overline{\mathfrak{Z}}^{\tilde{n}}}{\sqrt{R(n) R(\tilde{n})}} \frac{\mathfrak{W}^{m} \overline{\mathfrak{W}}^{\tilde{m}}}{\sqrt{R(m) R(\tilde{m})}} e^{-i \tau \tilde{E}_{n, m}} \\
& \times\left|\chi^{j}\right\rangle \otimes|\tilde{n}\rangle\langle\tilde{m}|\otimes| m\rangle\langle n|,
\end{aligned}
$$


where $\mathfrak{W}=\operatorname{diag}\left(w_{1}, w_{2}, w_{3}, w_{4}\right), w_{j}=\rho_{j} e^{i \varphi_{j}}$ with $\rho_{j} \geq 0, \varphi_{j} \in[0,2 \pi)$ and $R(m)=m ! \mathbb{I}_{4}$, forms a set of VCS on $\mathbb{C}^{4} \otimes \mathcal{H}_{q} \otimes \mathcal{H}_{q}$. These states satisfy a normalization condition to unity given by

$$
\sum_{j=1}^{4} \sum_{\tilde{n}, \tilde{m}=0}^{\infty}(\mathfrak{Z}, \mathfrak{W}, \tau, j, \tilde{n}, \tilde{m} \mid \mathfrak{Z}, \mathfrak{W}, \tau, j, \tilde{n}, \tilde{m})=1
$$

with

$$
\mathcal{N}(\mathfrak{Z}, \mathfrak{W})=e^{2\left(r_{1}^{2}+\rho_{1}^{2}\right)}+e^{2\left(r_{2}^{2}+\rho_{2}^{2}\right)}+e^{2\left(r_{3}^{2}+\rho_{3}^{2}\right)}+e^{2\left(r_{4}^{2}+\rho_{4}^{2}\right)}
$$

Let $D=\left\{\left(z_{1}, z_{2}, z_{3}, z_{4}\right) \in \mathbb{C}^{4}|| z_{j} \mid<\infty, j=1,2,3,4\right\}, \mathcal{D}=\left\{\left(w_{1}, w_{2}, w_{3}, w_{4}\right) \in\right.$ $\left.\mathbb{C}^{4}|| w_{j} \mid<\infty, j=1,2,3,4\right\}$. Then, we have the following proposition.

Proposition 4.2. The VCS (4.12) satisfy on the quantum Hilbert space $\mathbb{C}^{4} \otimes \mathcal{H}_{q} \otimes \mathcal{H}_{q}$ a resolution of the identity as follows:

$$
\begin{aligned}
\sum_{j=1}^{4} \sum_{\tilde{m}=0}^{\infty} \sum_{\tilde{n}=0}^{\infty} \frac{1}{\tilde{m} ! \tilde{n} !} \\
\quad \times \int_{D \times \mathcal{D}} d \mu(\mathfrak{Z}, \mathfrak{W})\left(\overrightarrow{\partial_{\bar{z}_{j}}}\right)^{\tilde{n}}\left(\overrightarrow{\partial_{\bar{w}_{j}}}\right)^{\tilde{m}}[\mathcal{N}(\mathfrak{Z}, \mathfrak{W}) \mid \mathfrak{Z}, \mathfrak{W}, \tau, j, \tilde{n}, \tilde{m}) \\
\quad \times(\mathfrak{Z}, \mathfrak{W}, \tau, j, \tilde{n}, \tilde{m} \mid]\left(\overleftarrow{\partial_{z_{j}}}\right)^{\tilde{n}}\left(\overleftarrow{\partial_{w_{j}}}\right)^{\tilde{m}} \\
=\mathbb{I}_{4} \otimes \mathbb{I}_{q} \otimes \mathbb{I}_{q},
\end{aligned}
$$

where the measure $d \mu(\mathfrak{Z}, \mathfrak{W})$ is given on $D \times \mathcal{D}$ by

$$
d \mu(\mathfrak{Z}, \mathfrak{W})=\frac{1}{(2 \pi)^{8}} \prod_{j=1}^{4} \lambda\left(r_{j}\right) \varpi\left(\rho_{j}\right) d r_{j} d \rho_{j} d \theta_{j} d \varphi_{j} .
$$

Proof. In order to prove (4.15), let us first expand the integrand as

$$
\begin{aligned}
\sum_{j=1}^{4}\left(\overrightarrow{{\overrightarrow{z_{j}}}_{j}}\right)^{\tilde{n}}\left(\overrightarrow{\vec{\partial}_{\bar{w}_{j}}}\right)^{\tilde{m}}[\mathcal{N}(\mathfrak{Z}, \mathfrak{W}) \mid \mathfrak{Z}, \mathfrak{W}, \tau, j, \tilde{n}, \tilde{m})(\mathfrak{Z}, \mathfrak{W}, \tau, j, \tilde{n}, \tilde{m} \mid]\left(\overleftarrow{\partial_{z_{j}}}\right)^{\tilde{n}}\left(\overleftarrow{\partial_{w_{j}}}\right)^{\tilde{m}} \\
=\sum_{j=1}^{4} \sum_{n, n^{\prime}, m, m^{\prime}=0}^{\infty}\left(\overrightarrow{\partial_{\bar{z}_{j}}}\right)^{\tilde{n}}\left(\overrightarrow{\partial_{\bar{w}_{j}}}\right)^{\tilde{m}}\left(F_{n}(\mathfrak{Z}) F_{m}(\mathfrak{W})\left|\chi^{j}\right\rangle \otimes|\tilde{n}\rangle\langle\tilde{m}|\otimes| m\rangle\langle n|\right) \\
\quad \times\left(F_{n^{\prime}}(\mathfrak{Z}) F_{m^{\prime}}(\mathfrak{W})\left|\chi^{j}\right\rangle \otimes|\tilde{n}\rangle\left\langle\tilde{m}|\otimes| m^{\prime}\right\rangle\left\langle n^{\prime}\right|\right)^{\dagger}\left(\overleftarrow{\partial_{z_{j}}}\right)^{\tilde{n}}\left(\overleftarrow{\partial_{w_{j}}}\right)^{\tilde{m}} \\
:=\sum_{j=1}^{4} \sum_{n, n^{\prime}, m, m^{\prime}=0}^{\infty}\left[\left(\partial_{\bar{z}_{j}}\right)^{\tilde{n}} F_{n}(\mathfrak{Z})\left(\partial_{\bar{w}_{j}}\right)^{\tilde{m}} F_{m}(\mathfrak{W}) \mid \chi^{j}, \tilde{n}, \tilde{m}\right)\left(\chi^{j}, \tilde{n}, \tilde{m} \mid\right. \\
\left.\quad \times\left(\partial_{z_{j}}\right)^{\tilde{n}}\left(F_{n^{\prime}}(\mathfrak{Z})\right)^{*}\left(\partial_{w_{j}}\right)^{\tilde{m}}\left(F_{m^{\prime}}(\mathfrak{W})\right)^{*}\right] \otimes|m\rangle\left\langle n \mid n^{\prime}\right\rangle\left\langle m^{\prime}\right|
\end{aligned}
$$


call $\mathcal{I}$ the operator on the left-hand side of (4.15) and choose arbitrary vectors $\Psi, \Psi^{\prime}, \Phi, \Phi^{\prime}$ on the Hilbert space $\mathcal{H}_{q} \otimes \mathcal{H}_{q}$. Then, using (4.17), we have

$$
\begin{aligned}
\left(\Psi, \Psi^{\prime}|\mathcal{I}| \Phi, \Phi^{\prime}\right)= & \sum_{j=1}^{4} \sum_{\tilde{m}=0}^{\infty} \sum_{\tilde{n}=0}^{\infty} \frac{1}{\tilde{m} ! \tilde{n} !} \int_{D \times \mathcal{D}} d \mu(\mathfrak{Z}, \mathfrak{W}) \\
& \times \sum_{n, n^{\prime}, m, m^{\prime}=0}^{\infty}\left(\Psi \mid\left[\left(\partial_{\bar{z}_{j}}\right)^{\tilde{n}} F_{n}(\mathfrak{Z})\left(\partial_{\bar{w}_{j}}\right)^{\tilde{m}} F_{m}(\mathfrak{W}) \mid \chi^{j}, \tilde{n}, \tilde{m}\right)\right. \\
& \left.\times\left(\chi^{j}, \tilde{n}, \tilde{m} \mid\left(\partial_{z_{j}}\right)^{\tilde{n}}\left(F_{n^{\prime}}(\mathfrak{Z})\right)^{*}\left(\partial_{w_{j}}\right)^{\tilde{m}}\left(F_{m^{\prime}}(\mathfrak{W})\right)^{*}\right] \mid \Phi\right) \\
& \otimes\left(\Psi^{\prime}|m\rangle\left\langle n \mid n^{\prime}\right\rangle\left\langle m^{\prime}\right| \Phi^{\prime}\right) .
\end{aligned}
$$

The use of the boundedness of the operator $T$ and of the fact that $\sum_{j=1}^{4}\left|\chi^{j}\right\rangle\left\langle\chi^{j}\right|=\mathbb{I}_{4}$ allows to interchange the sum over $j$ with the integral and the four sums over $n, n^{\prime}$ and $m, m^{\prime}$, respectively. Thus,

$$
\begin{aligned}
& \left(\Psi, \Psi^{\prime}|\mathcal{I}| \Phi, \Phi^{\prime}\right)=\sum_{\tilde{m}=0}^{\infty} \sum_{\tilde{n}=0}^{\infty} \frac{1}{\tilde{m} ! \tilde{n} !} \int_{D \times \mathcal{D}} d \mu(\mathfrak{Z}, \mathfrak{W}) \\
& \times \sum_{n, n^{\prime}, m, m^{\prime}=0}^{\infty}\left[\sum _ { j = 1 } ^ { 4 } \left(\Psi | \chi ^ { j } \rangle [ ( \partial _ { \overline { z } _ { j } } ) ^ { \tilde { n } } F _ { n } ( \mathfrak { Z } ) ( \partial _ { \overline { w } _ { j } } ) ^ { \tilde { m } } F _ { m } ( \mathfrak { W } ) | \tilde { n } , \tilde { m } ) \left(\tilde{n}, \tilde{m} \mid\left(\partial_{z_{j}}\right)^{\tilde{n}}\right.\right.\right. \\
& \left.\left.\times\left(F_{n^{\prime}}(\mathfrak{Z})\right)^{*}\left(\partial_{w_{j}}\right)^{\tilde{m}}\left(F_{m^{\prime}}(\mathfrak{W})\right)^{*}\right]\left\langle\chi^{j}\right| \Phi\right)[] \otimes\left(\Psi^{\prime}|m\rangle\left\langle n \mid n^{\prime}\right\rangle\left\langle m^{\prime}\right| \Phi^{\prime}\right) \\
& =\sum_{\tilde{m}=0}^{\infty} \sum_{\tilde{n}=0}^{\infty} \frac{1}{\tilde{m} ! \tilde{n} !} \int_{D \times \mathcal{D}} d \mu(\mathfrak{Z}, \mathfrak{W}) \\
& \times \sum_{n, n^{\prime}, m, m^{\prime}=0}^{\infty} \operatorname{diag}\left(\frac{\tilde{n} ! z_{1}^{n}}{\sqrt{n ! \tilde{n} !}} \frac{\tilde{m} ! w_{1}^{m}}{\sqrt{m ! \tilde{m} !}}, \ldots, \frac{\tilde{n} ! z_{4}^{n}}{\sqrt{n ! \tilde{n} !}} \frac{\tilde{m} ! w_{4}^{m}}{\sqrt{m ! \tilde{m} !}}\right) \\
& \times \operatorname{diag}\left(\frac{\tilde{n} ! \bar{z}_{1}^{n^{\prime}}}{\sqrt{n^{\prime} ! \tilde{n} !}} \frac{\tilde{m} ! \bar{w}_{1}^{m^{\prime}}}{\sqrt{m^{\prime} ! \tilde{m} !}}, \ldots, \frac{\tilde{n} ! \bar{z}_{4}^{n^{\prime}}}{\sqrt{n^{\prime} ! \tilde{n} !}} \frac{\tilde{m} ! \bar{w}_{4}^{m^{\prime}}}{\sqrt{m^{\prime} ! \tilde{m} !}}\right) \\
& \times(\Psi \mid \tilde{n}, \tilde{m})(\tilde{n}, \tilde{m} \mid \Phi) \otimes\left(\Psi^{\prime} \mid n^{\prime}, m^{\prime}\right)\left(n, m \mid \Phi^{\prime}\right) \\
& =\sum_{m, \tilde{m}=0}^{\infty} \sum_{n, \tilde{n}=0}^{\infty}\left(\Psi \mid \operatorname{diag}\left(2 \pi \int_{0}^{\infty} r_{1} d r_{1} W\left(r_{1}\right) \frac{r_{1}^{2 n}}{n !} 2 \pi\right.\right. \\
& \times \int_{0}^{\infty} \rho_{1} d \rho_{1} \tilde{W}\left(\rho_{1}\right) \frac{\rho_{1}^{2 m}}{m !}, \ldots, 2 \pi \int_{0}^{\infty} r_{4} d r_{4} W\left(r_{4}\right) \frac{r_{4}^{2 n}}{n !} 2 \pi \\
& \left.\left.\times \int_{0}^{\infty} \rho_{4} d \rho_{4} \tilde{W}\left(\rho_{4}\right) \frac{\rho_{4}^{2 m}}{m !}\right)\langle\tilde{n} \mid \tilde{n}\rangle\langle\tilde{m} \mid \tilde{m}\rangle \mid \Phi\right) \otimes\left(\Psi^{\prime}|\langle n \mid n\rangle\langle m \mid m\rangle| \Phi^{\prime}\right) \\
& =\left(\Psi, \Psi^{\prime} \mid \Phi, \Phi^{\prime}\right),
\end{aligned}
$$


where the moment problems are solved by $W\left(r_{j}\right)=(1 / 2 \pi) \lambda\left(r_{j}\right), \tilde{W}\left(\rho_{j}\right)=(1 / 2 \pi) \varpi\left(\rho_{j}\right)$ with $\lambda\left(r_{j}\right)=2 e^{-r_{j}^{2}}, \varpi\left(\rho_{j}\right)=2 e^{-\rho_{j}^{2}}$.

Besides, we have the following proposition.

Proposition 4.3. These states fulfill the following properties:

(i) Temporal stability

$$
\mathbb{U}(t) \mid \mathfrak{Z}, \mathfrak{W}, \tau, j, \tilde{n}, \tilde{m})=\mid \mathfrak{Z}, \mathfrak{W}, \tau+t, j, \tilde{n}, \tilde{m}), \quad \mathbb{U}(t)=e^{-i t \mathbb{H}^{\mathrm{dim}}}
$$

(ii) Action identity

$$
\begin{gathered}
\sum_{j=1}^{4} \sum_{\tilde{n}, \tilde{m}=0}^{\infty}\left(\mathfrak{Z}, \mathfrak{W}, \tau, j, \tilde{n}, \tilde{m}\left|\mathbb{H}^{\operatorname{dim}}\right| \mathfrak{Z}, \mathfrak{W}, \tau, j, \tilde{n}, \tilde{m}\right) \\
=\frac{\tilde{\Omega}_{+}}{\tilde{\Omega}}|\mathfrak{Z}|^{2}+\frac{\tilde{\Omega}_{-}}{\tilde{\Omega}}|\mathfrak{W}|^{2}+\frac{1}{2}
\end{gathered}
$$

\subsection{Quaternionic vector coherent states}

\subsubsection{Construction}

We briefly discuss now the QVCS construction and their connection with the studied VCS. In $(4.12)$, set $\mathfrak{Z}=\operatorname{diag}(z, \bar{z}, z, \bar{z})$ and $\mathfrak{W}=\operatorname{diag}(w, \bar{w}, w, \bar{w})$ where $z=r e^{-i \tilde{\phi}}, w=\rho e^{-i \tilde{\varphi}}$ with $r, \rho \geq 0, \tilde{\phi}, \tilde{\varphi} \in[0,2 \pi)$. Consider $u, v \in \mathrm{SU}(2)$ and take $\mathcal{Z}=U \mathfrak{Z} U^{\dagger}, \mathcal{W}=V \mathfrak{W} V^{\dagger}$ where $U=\operatorname{diag}(u, u), V=\operatorname{diag}(v, v)$. Introduce the quaternions $\mathfrak{q}=A(r) e^{i \vartheta \Theta(\hat{n})}, \mathfrak{Q}=$ $B(\rho) e^{i \gamma \tilde{\Theta}(\hat{k})}$ with $\Theta(\hat{n})=\operatorname{diag}(\sigma(\hat{n}), \sigma(\hat{n})), \tilde{\Theta}(\hat{k})=\operatorname{diag}(\tilde{\sigma}(\hat{k}), \tilde{\sigma}(\hat{k}))$, where $A(r)=r \mathbb{I}_{4}$, $B(\rho)=\rho \mathbb{I}_{4}$ and

$$
\sigma(\hat{n})=\left(\begin{array}{rr}
\cos \phi & e^{i \eta} \sin \phi \\
e^{-i \eta} \sin \phi & -\cos \phi
\end{array}\right), \quad \tilde{\sigma}(\hat{k})=\left(\begin{array}{cc}
\cos \varphi & e^{i \varrho} \sin \varphi \\
e^{-i \varrho} \sin \varphi & -\cos \varphi
\end{array}\right)
$$

where $\phi, \varphi \in[0, \pi]$ and $\vartheta, \gamma, \eta, \varrho \in[0,2 \pi)$.

From the scheme developed in [54], since $u, v$ are given as $u=u_{\xi_{1}} u_{\phi_{1}} u_{\xi_{2}}, v=v_{\zeta_{1}} v_{\phi_{2}} v_{\zeta_{2}}$ with $u_{\xi_{1}}=\operatorname{diag}\left(e^{i \xi_{1} / 2}, e^{-i \xi_{1} / 2}\right), u_{\xi_{2}}=\operatorname{diag}\left(e^{i \xi_{2} / 2}, e^{-i \xi_{2} / 2}\right), v_{\zeta_{1}}=\operatorname{diag}\left(e^{i \zeta_{1} / 2}, e^{-i \zeta_{1} / 2}\right), v_{\zeta_{2}}=$ $\operatorname{diag}\left(e^{i \zeta_{2} / 2}, e^{-i \zeta_{2} / 2}\right)$, and

$$
u_{\phi_{1}}=\left(\begin{array}{cc}
\cos \frac{\phi_{1}}{2} & i \sin \frac{\phi_{1}}{2} \\
i \sin \frac{\phi_{1}}{2} & \cos \frac{\phi_{1}}{2}
\end{array}\right), \quad v_{\phi_{2}}=\left(\begin{array}{cc}
\cos \frac{\phi_{2}}{2} & i \sin \frac{\phi_{2}}{2} \\
i \sin \frac{\phi_{2}}{2} & \cos \frac{\phi_{2}}{2}
\end{array}\right), \quad \xi_{1}, \xi_{2}, \zeta_{1}, \zeta_{2} \in[0,2 \pi)
$$

for $\xi_{1}=\xi_{2}=\eta$ and $\zeta_{1}=\zeta_{2}=\varrho$, we get the following identifications: $\mathcal{Z}=r\left(\mathbb{I}_{4} \cos \vartheta+\right.$ $i \Theta(\hat{n}) \sin \vartheta)=\mathfrak{q}, \mathcal{W}=\rho\left(\mathbb{I}_{4} \cos \gamma+i \tilde{\Theta}(\hat{k}) \sin \gamma\right)=\mathfrak{Q}$. 
Then, the QVCS obtained as $\left.\left.\mid U \mathfrak{Z} U^{\dagger}, V \mathfrak{W} V^{\dagger}, \tau, j, \tilde{n}, \tilde{m}\right)=\mid \mathfrak{q}, \mathfrak{Q}, \tau, j, \tilde{n}, \tilde{m}\right)$ can be written as

$$
\begin{aligned}
\mid \mathfrak{q}, \mathfrak{Q}, \tau, j, \tilde{n}, \tilde{m})= & (\mathcal{N}(r, \rho))^{-1 / 2} \sum_{n, m=0}^{\infty} \frac{\mathfrak{q}^{n} \overline{\mathfrak{q}}^{\tilde{n}}}{\sqrt{n ! \tilde{n} !}} \frac{\mathfrak{Q}^{m} \overline{\mathfrak{Q}}^{\tilde{m}}}{\sqrt{m ! \tilde{m} !}} \\
& \times e^{-i \tau \tilde{E}_{n, m}}\left|\chi^{j}\right\rangle \otimes|\tilde{n}\rangle\langle\tilde{m}|\otimes| m\rangle\langle n|
\end{aligned}
$$

They satisfy a normalization condition to unity given by

$$
\sum_{j=1}^{4} \sum_{\tilde{n}, \tilde{m}=0}^{\infty}(\mathfrak{q}, \mathfrak{Q}, \tau, j, \tilde{n}, \tilde{m} \mid \mathfrak{q}, \mathfrak{Q}, \tau, j, \tilde{n}, \tilde{m})=1
$$

which provides $\mathcal{N}(r, \rho)=4 e^{2\left(r^{2}+\rho^{2}\right)}$.

Proposition 4.4. The QVCS (4.24) fulfill a resolution of the identity property on $\mathbb{C}^{4} \otimes$ $\mathcal{H}_{q} \otimes \mathcal{H}_{q}$ given by

$$
\begin{aligned}
\sum_{j=1}^{4} & \sum_{\tilde{m}=0}^{\infty} \sum_{\tilde{n}=0}^{\infty} \frac{1}{\tilde{m} ! \tilde{n} !} \int_{D_{1} \times D_{2}} d \mu(\mathfrak{q}, \mathfrak{Q})\left(\overrightarrow{\partial_{r}}\right)^{\tilde{n}}\left(\overrightarrow{\partial_{\rho}}\right)^{\tilde{m}} \\
& \times[W(r, \rho) \mid \mathfrak{q}, \mathfrak{Q}, \tau, j, \tilde{n}, \tilde{m})(\mathfrak{q}, \mathfrak{Q}, \tau, j, \tilde{n}, \tilde{m} \mid]\left(\overleftarrow{\partial_{r}}\right)^{\tilde{n}}\left(\overleftarrow{\partial_{\rho}}\right)^{\tilde{m}} \\
= & \mathbb{I}_{4} \otimes \mathbb{I}_{q} \otimes \mathbb{I}_{q}
\end{aligned}
$$

where $d \mu(\mathfrak{q}, \mathfrak{Q})=\frac{1}{16 \pi^{2}} r d r \rho d \rho(\sin \phi) d \phi d \eta d \vartheta(\sin \varphi) d \varphi d \varrho d \gamma$ on $D_{1} \times D_{2}$;

$D_{1}=\{(r, \phi, \eta, \vartheta) \mid 0 \leq r<\infty, 0 \leq \phi \leq \pi, 0 \leq \eta, \vartheta<2 \pi\}$ and $D_{2}=\{(\rho, \varphi, \varrho, \gamma) \mid 0 \leq \rho<$ $\infty, 0 \leq \varphi \leq \pi, 0 \leq \varrho, \gamma<2 \pi\}$.

The moment problem issued from (4.26), stated as follows:

$$
\int_{0}^{\infty} \int_{0}^{\infty} \frac{4 \pi^{2} W(r, \rho)}{\mathcal{N}(r, \rho)} \frac{r^{2 n}}{n !} \frac{\rho^{2 m}}{m !} r d r \rho d \rho=1
$$

is solved with

$$
W(r, \rho)=\frac{1}{\pi^{2}} \mathcal{N}(r, \rho) e^{-\left(r^{2}+\rho^{2}\right)} .
$$

A connection with the Weyl-Heisenberg group is realized by considering the unitary operators given by

$$
\begin{gathered}
U_{R}(0, \mathfrak{q})=e^{\left[-\mathfrak{q} \otimes a_{R}^{\dagger}+\mathfrak{q}^{\dagger} \otimes a_{R}\right]}=e^{-1 / 2\left[-\mathfrak{q} \otimes a_{R}^{\dagger}, \mathfrak{q}^{\dagger} \otimes a_{R}\right]} e^{\mathfrak{q}^{\dagger} \otimes a_{R}} e^{-\mathfrak{q} \otimes a_{R}^{\dagger}}, \\
U_{L}(0, \mathfrak{Q})=e^{\left[\mathfrak{Q} \otimes d_{L}^{\dagger}-\mathfrak{Q}^{\dagger} \otimes d_{L}\right]}=e^{-1 / 2\left[\mathfrak{Q} \otimes d_{L}^{\dagger},-\mathfrak{Q}^{\dagger} \otimes d_{L}\right]} e^{\mathfrak{Q} \otimes d_{L}^{\dagger}} e^{-\mathfrak{Q}^{\dagger} \otimes d_{L}},
\end{gathered}
$$

such that

$$
\begin{aligned}
\mid \mathfrak{q}, \mathfrak{Q}, \tau, j, \tilde{n}, \tilde{m})= & \frac{e^{-\left(r^{2}+\rho^{2}\right) / 2}}{2} \mathbb{U}(\tau) \\
& \times\left[\frac{\overline{\mathfrak{q}}^{\tilde{n}} \overline{\mathfrak{Q}}^{\tilde{m}}}{\sqrt{\tilde{n} ! \tilde{m} !}}\left|\chi^{j}\right\rangle \otimes|\tilde{n}\rangle\left\langle\tilde{m}\left|\otimes U_{L}(0, \mathfrak{Q})\right| 0\right\rangle\langle 0| U_{R}(0, \mathfrak{q})\right],
\end{aligned}
$$


where the operators $a_{R}$ and $d_{L}$ act on a given state $|\tilde{n}\rangle\langle\tilde{m}|\otimes| m\rangle\langle n|$ as follows:

$$
\begin{aligned}
a_{R}|\tilde{n}\rangle\langle\tilde{m}|\otimes| m\rangle\langle n| & :=|\tilde{n}\rangle\langle\tilde{m}|\otimes| m\rangle\langle n| a \\
& =\sqrt{n+1}|\tilde{n}\rangle\langle\tilde{m}|\otimes| m\rangle\langle n+1| \\
d_{L}|\tilde{n}\rangle\langle\tilde{m}|\otimes| m\rangle\langle n| & :=|\tilde{n}\rangle\langle\tilde{m}|\otimes d| m\rangle\langle n| \\
& =\sqrt{m}|\tilde{n}\rangle\langle\tilde{m}|\otimes| m-1\rangle\langle n| .
\end{aligned}
$$

\subsubsection{QVCS statistical properties}

This part of the work deals with the physical features of the QVCS. The expectation which can be interpreted as the average of the observable that would be expected to obtain from a large number of measurements, and the dispersion of the quadrature operators on the Hilbert space $\mathbb{C}^{4} \otimes \mathcal{H}_{q} \otimes \mathcal{H}_{q}$ are investigated.

Let us consider the operators given on $\mathbb{C}^{4} \otimes \mathcal{H}_{q} \otimes \mathcal{H}_{q}$ by

$$
\begin{aligned}
\hat{P}_{X} & =\mathbb{I}_{4} \otimes \frac{-i \hbar}{\sqrt{2 \theta}}\left[a_{R}-a_{R}^{\dagger}, \cdot\right], \quad \hat{P}_{Y}=\mathbb{I}_{4} \otimes \frac{-\hbar}{\sqrt{2 \theta}}\left[a_{R}+a_{R}^{\dagger}, \cdot\right] \\
\hat{X} & =\mathbb{I}_{4} \otimes \sqrt{\frac{\theta}{2}}\left[a_{R}+a_{R}^{\dagger}\right], \quad \hat{Y}=\mathbb{I}_{4} \otimes i \sqrt{\frac{\theta}{2}}\left[a_{R}^{\dagger}-a_{R}\right] .
\end{aligned}
$$

From (4.31), we obtain

$$
\begin{aligned}
{\left[a_{R}-a_{R}^{\dagger},|\tilde{n}\rangle\langle\tilde{m}|\otimes| m\rangle\langle n|\right]=} & \sqrt{n+1}|\tilde{n}\rangle\langle\tilde{m}|\otimes| m\rangle\langle n+1| \\
& -\sqrt{n}|\tilde{n}\rangle\langle\tilde{m}|\otimes| m\rangle\langle n| .
\end{aligned}
$$

Denote the expectation value of an operator by $\langle\cdot\rangle=\sum_{\tilde{n}, \tilde{m}=0}^{\infty}(\mathfrak{q}, \mathfrak{Q}, j, \tilde{n}, \tilde{m}|\cdot| \mathfrak{q}, \mathfrak{Q}, j, \tilde{n}, \tilde{m})$. We get the following expressions:

$$
\begin{aligned}
& \left\langle\hat{P}_{X}\right\rangle= \pm \frac{\hbar}{2 \sqrt{2 \theta}} r \cos (\phi) \sin (\eta), \quad\left\langle\hat{P}_{X}^{2}\right\rangle=\frac{\hbar^{2}}{2 \theta}\left[r^{2} \sin ^{2}(\eta)+\frac{1}{4}\right], \\
& \left\langle\hat{P}_{Y}\right\rangle=-\frac{\hbar}{2 \sqrt{2 \theta}}[r \cos (\eta)], \quad\left\langle\hat{P}_{Y}^{2}\right\rangle=\frac{\hbar^{2}}{2 \theta}\left[r^{2} \cos ^{2}(\eta)+\frac{1}{4}\right]
\end{aligned}
$$

from which result the relations

$$
\begin{aligned}
& \left(\Delta \hat{P}_{X}\right)^{2}=\frac{1}{4}\left(\frac{\hbar^{2}}{2 \theta}\right)\left[4 r^{2} \sin ^{2}(\eta)-r^{2} \cos ^{2}(\phi) \sin ^{2}(\eta)+1\right] \\
& \left(\Delta \hat{P}_{Y}\right)^{2}=\frac{1}{4}\left(\frac{\hbar^{2}}{2 \theta}\right)\left[3 r^{2} \cos ^{2}(\eta)+1\right] .
\end{aligned}
$$


In the same way, we obtain

$$
\begin{aligned}
& (\Delta \hat{Y})^{2}=\frac{1}{4}\left(\frac{\theta}{2}\right)\left[4 r^{2} \sin ^{2}(\eta)-r^{2} \cos ^{2}(\phi) \sin ^{2}(\eta)+1\right] \\
& (\Delta \hat{X})^{2}=\frac{1}{4}\left(\frac{\theta}{2}\right)\left[3 r^{2} \cos ^{2}(\eta)+1\right]
\end{aligned}
$$

and the following uncertainties

$$
\begin{aligned}
& {[\Delta \hat{X} \Delta \hat{Y}]^{2} }=\frac{1}{16}\left(\frac{\theta^{2}}{4}\right) F(r, \eta, \phi)=\frac{1}{16}\left[\frac{1}{4}|\langle[\hat{X}, \hat{Y}]\rangle|^{2}\right] F(r, \eta, \phi), \\
& {\left[\Delta \hat{X} \Delta \hat{P}_{X}\right]^{2}=\frac{1}{16}\left(\frac{\hbar^{2}}{4}\right) F(r, \eta, \phi) \geq \frac{1}{16}\left[\frac{1}{4}\left|\left\langle\left[\hat{X}, \hat{P}_{X}\right]\right\rangle\right|^{2}\right], } \\
& {\left[\Delta \hat{Y} \Delta \hat{P}_{Y}\right]^{2}=\frac{1}{16}\left(\frac{\hbar^{2}}{4}\right) F(r, \eta, \phi) \geq \frac{1}{16}\left[\frac{1}{4}\left|\left\langle\left[\hat{Y}, \hat{P}_{Y}\right]\right\rangle\right|^{2}\right], } \\
& {\left[\Delta \hat{P}_{X} \Delta \hat{P}_{Y}\right]^{2}=\frac{1}{16}\left(\frac{\hbar^{4}}{4 \theta^{2}}\right) F(r, \eta, \phi) \geq \frac{1}{16}\left[\frac{1}{4}\left|\left\langle\left[\hat{P}_{X}, \hat{P}_{Y}\right]\right\rangle\right|^{2}\right]=0, }
\end{aligned}
$$

where

$$
F(r, \eta, \phi)=\left[3 r^{2} \cos ^{2}(\eta)+1\right]\left[4 r^{2} \sin ^{2}(\eta)-r^{2} \cos ^{2}(\phi) \sin ^{2}(\eta)+1\right] .
$$

Remark 4.1. As a matter of result checking, let us attract the reader attention on the fact that, in [23], a factor " 2 " has been forgotten in the author expressions (4.35)-(4.37) obtained for the dispersions of the momentum operators; one should read, in the denominator, the quantity $\hbar^{2} / 2 \theta$ instead of $\hbar^{2} / \theta$. Furthermore, in the mentioned work, a sign "-" should be also added in the expression of the operator $\hat{P}_{Y}$.

\section{Concluding Remarks}

A matrix formulation of a Hamiltonian describing the motion of an electron in an electromagnetic field with a confining harmonic potential in a two-dimensional noncommutative space has been provided in this work. Relevant thermodynamical and statistical properties of the physical system have been studied and discussed. In this analysis, some $\theta$-modified quantities have been obtained. In the limit $\theta \rightarrow 0$, these quantities can be identified with those derived in the commutative context related to the standard Landau problem. Then, the Matrix Vector Coherent States (MVCS) have been constructed and analyzed with respect to required properties. Besides, the QVCS as well as their connection with the VCS and their statistical properties have been investigated and discussed.

To conclude, let us mention that the physical model investigated in this work could be re-parameterized by the following operators

$$
\hat{q}_{1}=\hat{X}, \quad \hat{q}_{2}=\hat{Y}-\frac{\theta}{\hbar} \hat{P}_{X}, \quad \hat{p}_{1}=\hat{P}_{X}, \quad \hat{p}_{2}=\hat{P}_{Y}
$$

satisfying usual canonical commutation relations, and then be solved in terms of ordinary square-integrable functions $\psi\left(q_{1}, q_{2}\right)$. Such an investigation will be in the core of a forthcoming paper. 


\section{Acknowledgments}

This work was partially supported by the Abdus Salam International Centre for Theoretical Physics (ICTP, Trieste, Italy) through the Office of External Activities (OEA) - Prj-15. The ICMPA is in partnership with the Daniel Iagolnitzer Foundation (DIF), France.

\section{References}

[1] G. Alexanian, A. P. Balachandran, G. Immirzi and B. Ydri, Fuzzy $\mathbb{C} P^{2}$, preprint (2001), arXiv:hep-th/0103023.

[2] S. T. Ali, M. Engliš and J.-P. Gazeau, Vector coherent states from Plancherel's theorem, Clifford algebras and matrix domains, J. Phys. A: Math. Gen. 37 (2004) 6067-6089.

[3] P. D. Alvarez, J. Gomis, K. Kamimura and M. S. Plyushchay, Anisotropic harmonic oscillator, non-commutative Landau problem and exotic Newton-Hooke symmetry, Phys. Lett. B 659 (2008) 906, arXiv:0711.2644.

[4] A. P. Balachandran, K. S. Gupta and S. Kürkçüoğlu, Edge currents in non-commutative ChernSimons theory from a new matrix model, preprint (2003), arXiv:hep-th/0306255.

[5] S. D. Barlett, D. J. Rowe and J. Repka, Vector coherent state representations, induced representations and geometric quantization: I. Scalar coherent state representations, J. Phys. A: Math. Gen. 35 (2002) 5599.

[6] S. D. Barlett, D. J. Rowe and J. Repka, Vector coherent state representations, induced representations and geometric quantization: II. Vector coherent state representations, J. Phys. A: Math. Gen. 35 (2002) 5625.

[7] A. Cappelli, C. A. Trugenberger and G. R. Zemba, Infinite symmetry in the quantum hall effect, Nucl. Phys. B 396 (1993) 465.

[8] A. Connes, M. R. Douglas and A. Schwarz, Noncommutative geometry and matrix theory: Compactification on tori, J. High Energy Phys. 9802 (1998) 003.

[9] Ö. F. Dayi and A. Jellal, Landau diamagnetism in noncommutative space and the nonextensive thermodynamics of Tsallis, Phys. Lett. A 287 (2001) 349-355.

[10] Ö. F. Dayi and A. Jellal, Hall effect in noncommutative coordinates, J. Math. Phys. 43 (2002) 4592.

[11] S. Doplicher, K. Fredenhagen and J. E. Roberts, The quantum structure of spacetime at the planck scale and quantum fields, Comm. Math. Phys. 172 (1995) 187.

[12] M. R. Douglas and N. A. Nekrasov, Noncommutative field theory, Rev. Mod. Phys. 73 (2001) 977.

[13] S. Dulat and K. Li, Landau problem in noncommutative quantum mechanics, Chin. Phys. C 32 (2008) 92.

[14] G. Dunne and R. Jackiw, "Peierls substitution" and Chern-Simons quantum mechanics, Nucl. Phys. B (Proc. Suppl.) 33C (1993) 114.

[15] G. Dunne, R. Jackiw and C. A. Trugenberger, Topological (Chern-Simons) quantum mechanics, Phys. Rev. D 41 (1990) 661.

[16] C. Duval and P. A. Horváthy, The "Peierls substitution" and the exotic Galilei group, Phys. Lett. B 479 (2000) 284.

[17] C. Duval and P. A. Horváthy, Exotic galilean symmetry in the non-commutative plane, and the Hall effect, J. Phys. A 34 (2001) 10097.

[18] D. H. Feng, J. R. Klauder and M. Strayer (eds.), Coherent States: Past, Present and Future (World Scientific, Singapore, 1994).

[19] J. Gamboa, M. Loewe, F. Méndez and J. C. Rojas, The Landau problem in noncommutative quantum mechanics, Mod. Phys. Lett. A 16 (2001) 2075, arXiv:hep-th/0104224.

[20] J.-P. Gazeau, P. Y. Hsiao and A. Jellal, A coherent-state approach to two-dimensional electron magnetism, Phys. Rev. B 65 (2002) 094427.

[21] J. B. Geloun, S. Gangopadhyay and F. G. Scholtz, Harmonic oscillator in a background magnetic field in noncommutative quantum phase-space, Europhys. Lett. 86 (2009) 51001. 
[22] J. B. Geloun, J. Govaerts and M. N. Hounkonnou, A $(p, q)$-deformed Landau problem in a spherical harmonic well: Spectrum and noncommuting coordinates, Europhys. Lett. 80 (2007) 30001.

[23] J. B. Geloun and F. G. Scholtz, Coherent states in noncommutative quantum mechanics, J. Math. Phys. 50 (2009) 043505.

[24] M. O. Goerbig and P. Lederer, Introduction to the quantum Hall effects, Lecture notes (2006).

[25] M. O. Goerbig, P. Lederer and C. M. Smith, Solides et liquides quantiques, dans les systèmes bidimensionnels d'électrons, "Images de la physique" magazine du CNRS (2005) 105.

[26] H. Grosse and P. Prešnajder, The construction on noncommutative manifolds using coherent states, Lett. Math. Phys. 28 (1993) 239.

[27] P. A. Horváthy, The non-commutative Landau problem, Ann. Phys. 299 (2002) 128.

[28] Y. Ishikawa and H. Fukuyama, Orbital magnetism and current distribution of two-dimensional electrons under confining potential, J. Phys. Soc. Jpn. 68 (1999) 2405-2413.

[29] R. Jackiw, Physical instances of noncommuting coordinates, preprint (2001), arXiv:hep-th/ 0110057.

[30] A. Jellal, $U_{q}[\operatorname{sl}(2)]$ quantum algebra in quantum Hall effect, Int. J. Theor. Phys. 38 (1999) 1905.

[31] A. Jellal, Degeneracy of the lowest Landau level and $s u_{q}(2)$ on the Poincaré half-plane, Acta Phys. Slovaca 50(2) (2000) 253.

[32] A. Jellal, Orbital magnetism of a two-dimensional noncommutative confined system, J. Phys. A: Math. Gen. 34 (2001) 10159.

[33] L. D. Landau, Diamagnetismus der Metalle, Z. Phys. 64 (1930) 629.

[34] L. D. Landau and E. M. Lifshitz, Statistical Physics, Part 1 (Pergamon, Oxford, 1980).

[35] N. Macris and S. Ouvry, Projection on higher Landau levels and non-commutative geometry, J. Phys. A: Math. Gen. 35 (2002) 4477.

[36] N. Macris and S. Ouvry, Projection on higher Landau levels and non-commutative geometry, J. Phys. A: Math. Gen. 35 (2002) 8883.

[37] G. M. Magro, Noncommuting coordinates in the Landau problem, preprint (2003) arXiv:quant$\mathrm{ph} / 0302001$.

[38] R. P. Malik, Hamiltonian and Lagrangian dynamics in a noncommutative space, Mod. Phys. Lett. A 18 (2003) 2795.

[39] A. Mikovic, N. C. Dias and J. N. Prata (ed.), Quantum gravity and noncommutative geometry, Mod. Phys. Lett. A 20 (2005) 1261.

[40] V. P. Nair and A. P. Polychronakos, Quantum mechanics on the noncommutative plane and sphere, Phys. Lett. B 505 (2001) 267.

[41] V. Pasquier, Quantum Hall effect and noncommutative geometry, Séminaire Poincaré X (2007) $1-14$.

[42] R. Peierls, Zur theorie des diamagnetismus von leitungselektronen, Z. Phys. 80 (1933) 763.

[43] M. Riccardi, Physical observables for noncommutative Landau levels, J. Phys. A: Math. Gen. 39 (2006) 4257.

[44] D. J. Rowe and J. Repka, Vector coherent state theory as a theory of induced representations, J. Math. Phys. 32 (1991) 2614.

[45] D. J. Rowe, G. Rosensteel and R. Gilmore, Vector coherent state representation theory, J. Math. Phys. 26 (1985) 2787.

[46] H.-T. Sato, Landau levels and quantum group, Mod. Phys. Lett. A 9 (1994) 451.

[47] F. G. Scholtz, B. Chakraborty, S. Gangopadhyay and J. Govaerts, Interaction and noncommutativity in quantum Hall systems, J. Phys. A: Math. Gen. 38 (2005) 9849.

[48] F. G. Scholtz, B. Chakraborty, S. Gangopadhyay and A. G. Hazra, Dual families of noncommutative quantum systems, Phys. Rev. D 71 (2005) 085005.

[49] F. G. Scholtz, B. Chakraborty, J. Govaerts and S. Vaidya, Spectrum of the non-commutative spherical well, J. Phys. A: Math. Theor. 40 (2007) 14581. 
[50] F. G. Scholtz, L. Gouba, A. Hafver and C. M. Rohwer, Formulation, interpretation and application of non-commutative quantum mechanics, J. Phys. A: Math. Theor. 42 (2009) 175303.

[51] F. G. Scholtz and J. Govaerts, Thermodynamics of a non-commutative fermion gas, J. Phys. A: Math. Theor. 41 (2008) 505003.

[52] N. Seiberg and E. Witten, String theory and noncommutative geometry, J. High Energy Phys. 9909 (1999) 032.

[53] L. Susskind, The quantum Hall fluid and non-commutative Chern-Simons theory, preprint (2001) arXiv:hep-th/0101029.

[54] K. Thirulogasanthar and S. T. Ali, A class of vector coherent states defined over matrix domains, J. Math. Phys. 44 (2003) 5070-5083.

[55] K. Thirulogasanthar, G. Honnouvo and A. Krzyżak, Multi-matrix vector coherent states, Ann. of Phys. 314 (2004) 119-144.

[56] P.-M. Zhang and P. A. Horváthy, Chiral decomposition in the non-commutative Landau problem, preprint (2012), arXiv:hep-th/1112.0409. 